\title{
Wearable System for Obstacle Detection and Human Assistance Using Ultrasonic Sensor Array
}

By

Ashish Patankar, BE Electronics Engineering

A Thesis

In

Electrical Engineering

Submitted to the Graduate Faculty

Of Texas Tech University in

Partial Fulfillment of

the Requirements for

the Degree of

Master of Sciences

Approved

Dr. Tooraj Nikoubin

Chair of the committee

Dr. Brian Nutter

Co-chair of the committee

Dr. Stephen Bayne

Member of the committee

Mark Sheridan

Dean of the Graduate School

<May 2016> 
Copyright 2016, Ashish Patankar 


\section{ACKNOWLEDGMENTS}

First of all I would like to thank my family members, friends and my committee members for their support and help. Without their cooperation, it was impossible for me to achieve this goal in my life.

I would like to thank my adviser, Dr. Nikoubin for his excellent and valuable guidance. He always kept me motivated to work hard and always guided me to pursue the excellence. He was so helpful and supportive for all the difficulties I faced during my research work. I thank him for being a great adviser throughout my master's coursework. The courses which I had taken under Dr. Nikoubin, "Introduction to VLSI Design", “Advanced Digital System Design", "Testing of Digital Systems" and "Microprocessor Architecture" have strengthened my knowledge in digital design, also they were very helpful during my thesis work.

I would like to thank Dr. Nutter and Dr. Bayne, for being there on my thesis defense committee. Your valuable guidance in writing this document and achieving my goals during this coursework is most important for me. You both have always been an inspiration for me.

I would like to thank all the faculty members and staff of our department, who helped me throughout my master's studies.

I would like to thank my flat mates, Viraj, Vikram, Nikhil Kadu, Pranav for their support and love. They always encouraged me to achieve my goals. I would also like to thank my friends Nikhil Patil, Swetha, Amol, and Maithili for their valuable support. You all have always motivated me for my work.

Most importantly, I would love to thank my family, my mother -Asha, my father- Bhaskar, my sister-Ashwini and Nikhil Bhagwat and my girlfriend Sukhada for their love and support in my all ups and downs in my life. Without you all, it was impossible for me to achieve all the good things in my life. Thank you once again! 


\section{TABLE OF CONTENTS}

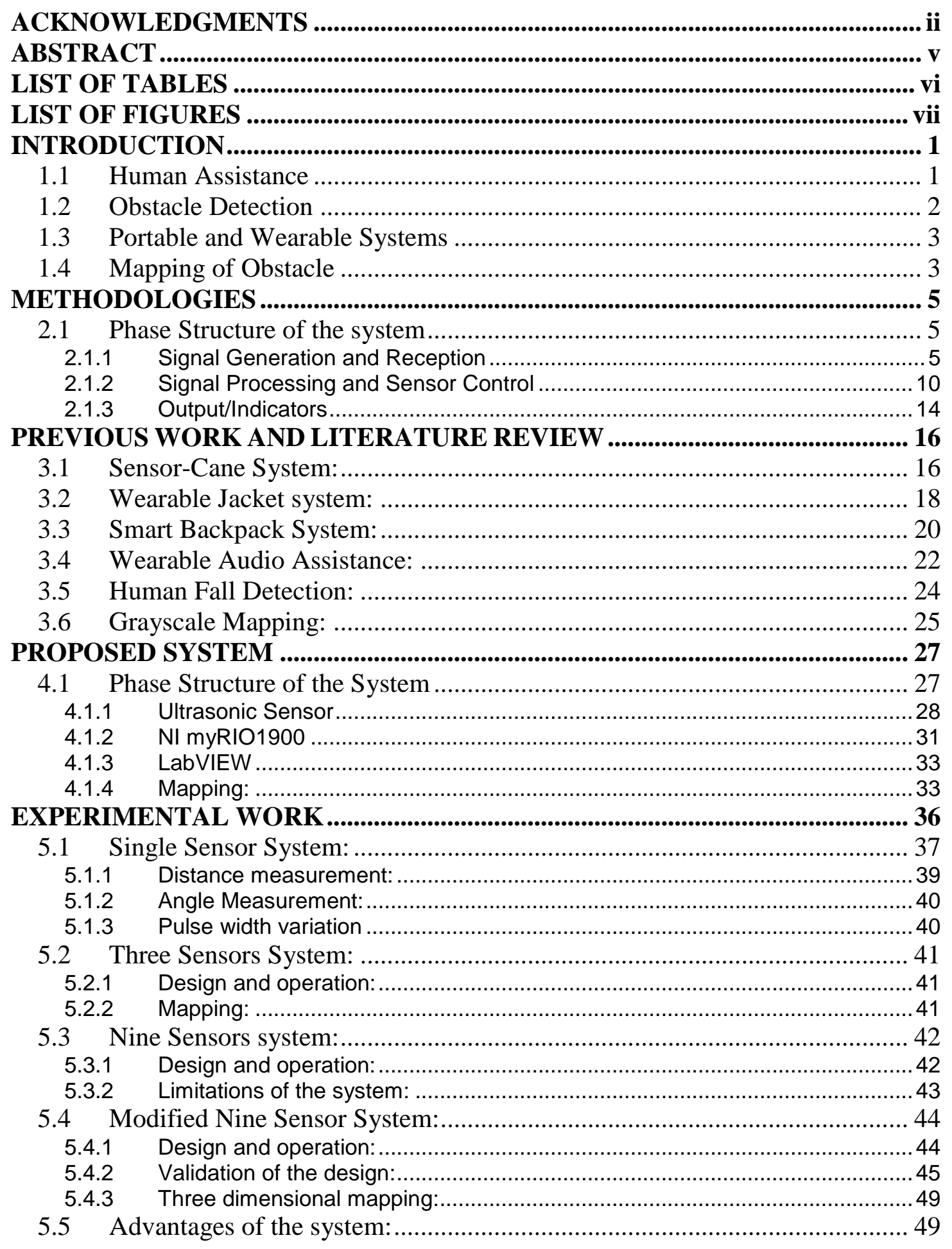




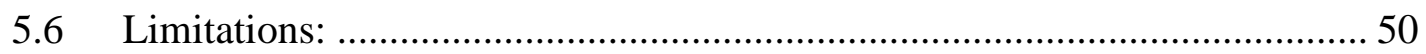

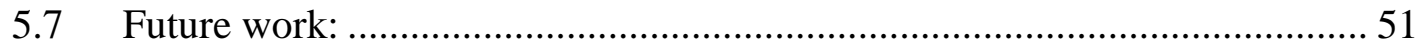

CONCLUSION

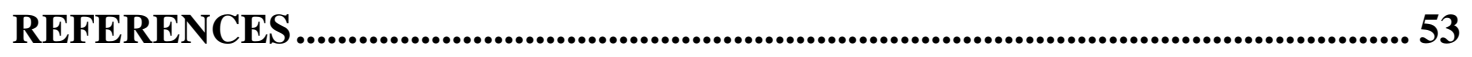




\begin{abstract}
Generally when we consider any system that deals with human assistance, basic requirements about the system are, it should be simple, user friendly and portable. As a person whoever is using this system need not be aware of all the operations which are taking place behind the screen, one must consider an ease of access to the user. Most of the human assistance systems are proposed to assist physically disabled people. In this project, we have proposed and tested a system which is based on the navigation and capable of proving information about the possible mapping of obstacles in surrounding.

System consists of three phases. First phase is associated with the signal generation of chirp signal and echoing. Ultrasonic sensor array is used for this phase of the system. Second phase is associated with the signal processing unit. National Instruments myRIO1900 which is a Reconfigurable-Input-Output device is used to access all the pins of sensors. This device is made portable by using external power supply and a Wi-Fi module. It can be accessed and programmed using LabVIEW platform. The third phase is the output phase. The output is represented in terms of $2 \mathrm{D}$ maps, generated using LabVIEW and 3D maps, and generated using MATLAB.

Design is proposed which is capable of providing some additional features in the system which makes it more reliable and can perform multiple tasks at the same time.
\end{abstract}




\section{LIST OF TABLES}

1. Comparison of different sensors 10

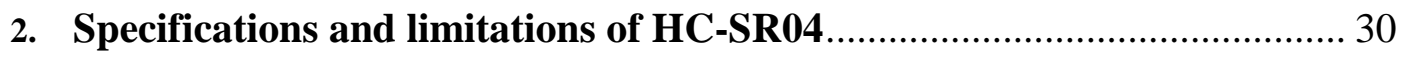

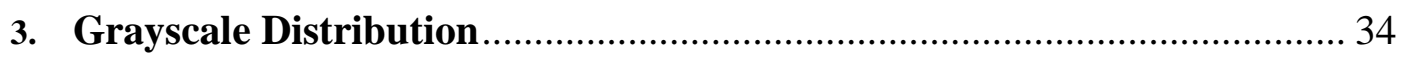

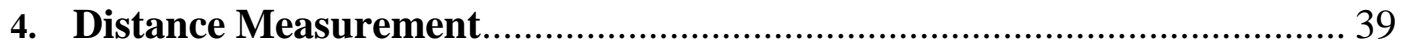




\section{LIST OF FIGURES}

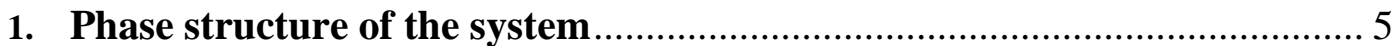

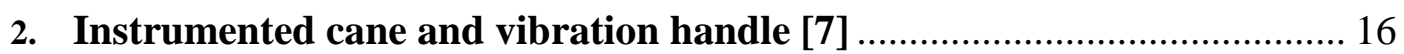

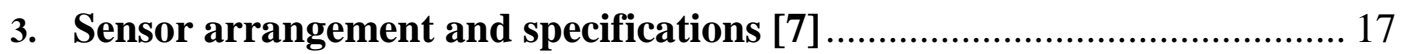

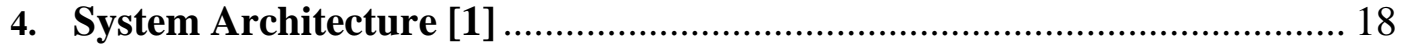

5. Layout and geometry of ultrasonic sensors [4] .................................... 21

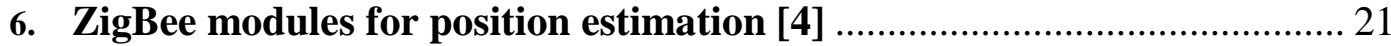

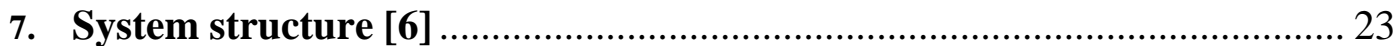

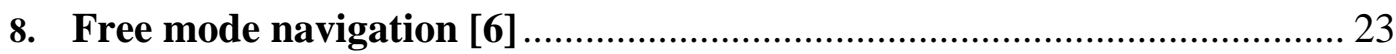

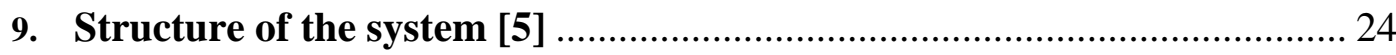

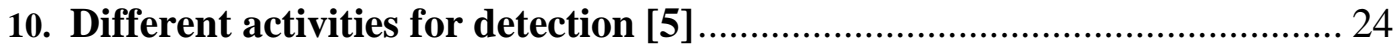

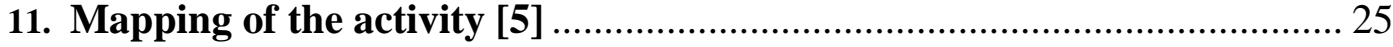

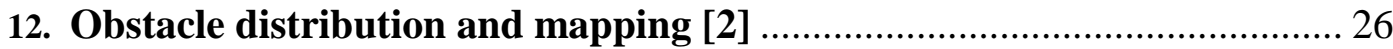

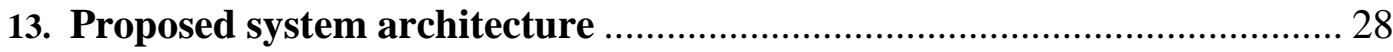

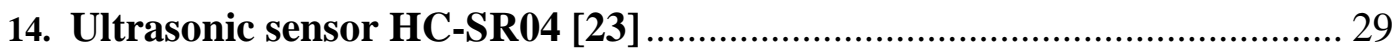

15. Testing of the performance for angular range [23] ..................................2 29

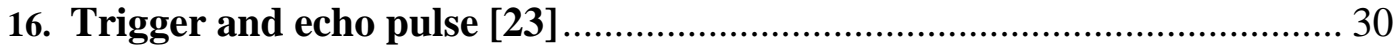

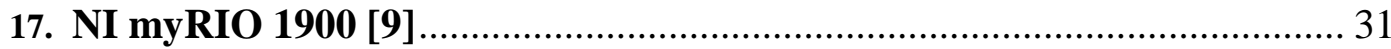

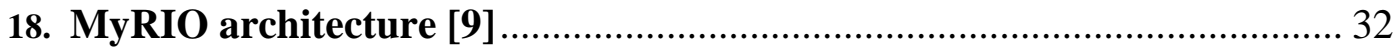

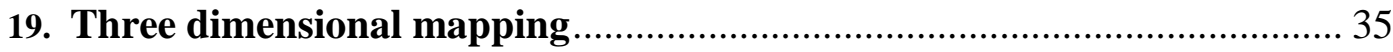

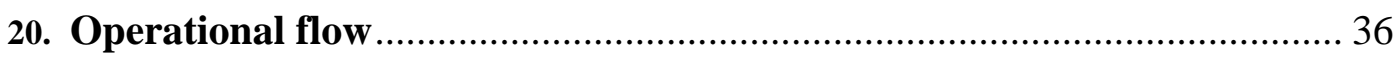

21. Experimental setup for Single Sensor System .................................. 38

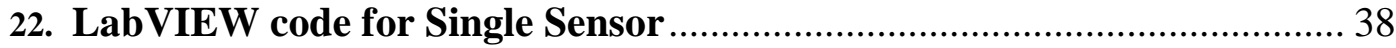

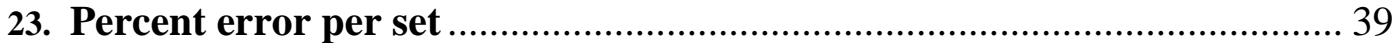

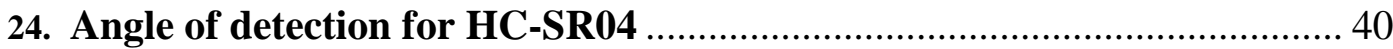

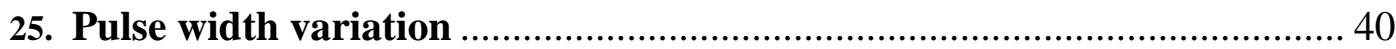

26. Three sensor system and mapping ................................................ 41

27. Nine sensor system and obstacle detection........................................ 42

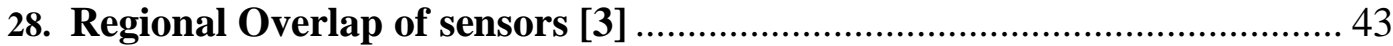

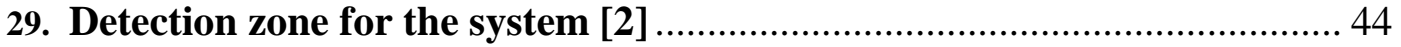




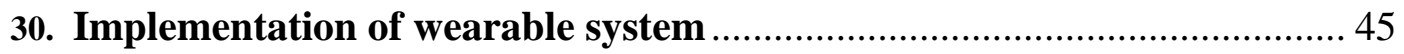

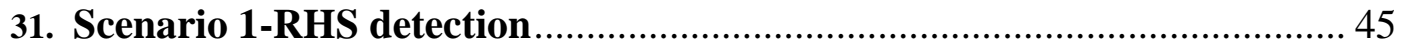

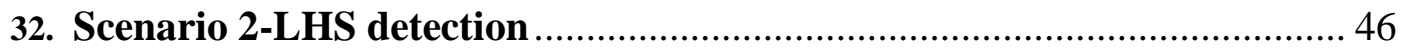

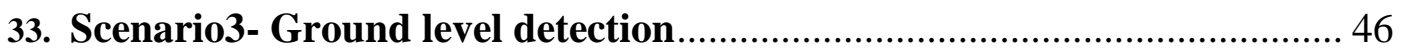

34. Scenario 4- walking state with multiple objects .......................................... 47

35. Scenario 5- walking state with LHS and center detection........................... 47

36. Scenario6- walking state object detection ................................................... 48

37. Scenario7- Walking state object detection ................................................... 48

38. Three dimensional mapping using projections ……................................... 49 


\section{CHAPTER I}

\section{INTRODUCTION}

In day-today life, while dealing with any kind of the system, one need not to know about each and every detail about it. It is sufficient for him to know the purpose behind the use of the system and how to use the system. But for the one who designs the system, it is needed to know each and every detail about it, including the background, functioning and debugging of it. If we think from designers approach, we should be able to answer the following questions,

1. Why do we need to do this?

2. What have we learned/studied so far?

3. What are we going to implement?

4. What types of results do we get?

In short, one must know the basic purpose behind it. He must have knowledge about the previous experiments which are carried out in the same area. He should implement his system, depending on the study he did so far. Last but not the least the results should be satisfactory and strong enough to make system reliable and robust.

\subsection{Human Assistance}

Our project is about the system, which is capable of detecting an obstacle and providing human assistance. The phrase "Human Assistance" deal with all the systems which are capable of providing an adequate help to the person whoever is using it. Most of the times, it is designed to perform tasks which are out of the reach of the person who is using it. The human and technology go with hand in hand. Technology effectively reduces the human efforts and ultimately improves the quality of life. We have different types of applications where we can use technology for human assistance. Healthcare is the biggest area where such systems are implemented. We often find people using wheelchair, walking assistance or audio-visual aids [12] [13]. In telecommunication, we use satellites, mobile phones. Military uses such systems in 
remote areas or in weapons. This area is popular in industries as well. The machinery which is used for manufacturing or other processes is automated for most of the times. Navigation is also an important field where the human assistance is needed.

In this project, we are going to cover two major areas which are very useful now-a-days. The basic purpose behind this project is about the healthcare. So our system is mainly focusing on the people who are visually impaired and need an aid to navigate in surrounding. Considering this purpose, we are mainly discussing about three major factors which cover the operation of the system.

\subsection{Obstacle Detection}

Obstacle can be termed as an object which comes across in a path of navigation. When a person is walking on his path, he needs to be aware of the objects which are in his path of navigation. Eyes are the most important organs of human body which gives us information about the objects which are at surroundings. They tell us about the color, nature of the obstacles. We can differentiate between the objects. When visual system of the human body fails to work, it becomes a challenge to navigate in unknown surrounding. It is quite difficult for the person to get an idea about all the stationary and moving obstacles around him. It is risky to rely on the noises coming from the surrounding. Hence obstacle detection becomes and important phase in our system.

In this project, our system is strictly focusing on the minimal hardware and data. This decreases the data that we are going to process. More is the data we are going to use, more is the hardware needed and more is the reliability of the system. Use of very less data may lead to the risky operation. Hence we need to make an optimization in between the complexity and reliability of the system. We are going to extract the prime objects from the surrounding which is mainly responsible for creating obstacles in a path of navigation. This will make system moderately reliable using lesser data. 


\subsection{Portable and Wearable Systems}

Portable technology is never the less a point of attraction for everyone. We often find people who carry portable systems with them. It can be anything like a mobile phone, digital camera, flash drive or a music player. Portable systems can be carried along with us anywhere we want. They provide an ease of access to the user. In order to add simplicity in functioning, the navigation systems for visually impaired people are necessary to be portable. They need to be light in weight so that the person can use them without facing any difficulty. It should be able to provide a user friendly operation for every user.

Wearable systems are subset of the portable systems. Wearable technology is becoming popular these days. We have plenty of examples to explain about the wearable systems. Now-a-days we see the watches includes camera or a phone device on it. A device that can keep statistical data about your health or glasses which are capable of scanning objects like your eyes do or earphone set which tells you about the map around you, all are categorized under the wearable system. In simple language, wearable system can be defined as the system that can be part of your daily wears. Like the daily wears, we should be able to wear the system on body without having any trouble. Considering the navigation system for the blind people, our system should be very compact, can be easily worn. The wearable system is going to play a key role in our project.

\subsection{Mapping of Obstacle}

As we discussed in 1.2, the obstacle detection is a difficult task for a blind person. We always need to think about the safety of the user. To analyze the surrounding with the help of very less information is always a tricky thing to do. Information we get by using any system is very small as compared to the information conveyed by eyes. Hence mapping of the obstacles is a critical step in the blind navigation system. It should be done is such a way that user should be able to analyze the most critical objects and create his own safe path. There are different types of methods available which are taking a closer approach to our aim. Maps can be a good 
solution to it. But again the main question is how to make user apprised of the incoming object? How to provide him accurate information about it? Audio devices can be of great use. They can give real time information about the situation. But they face some problems. Suppose you are walking on a road with a lot of noises from the surrounding. This is going to make a lot of problems for the user. He cannot get the accurate information about the obstacles because of the interferences. The other option can be use of the vibration motors over the body. But a single system is not sufficient to sketch the entire scenario in the users mind. Hence we need to use a combination of all the methods which will provide user a near most information about the object around him. 


\section{CHAPTER II}

\section{METHODOLOGIES}

Before we start to discuss about entire systems, let's have a review about popular ways to achieve our goals. Obviously we can find a large number of ways to do certain task. Depending upon the comparison between all the methods and our requirement, we will choose the components which are needed. We have also discussed about the nature of our system.

\subsection{Phase Structure of the system}

The system can be categorized in three phases depending on the functionality of the each block.

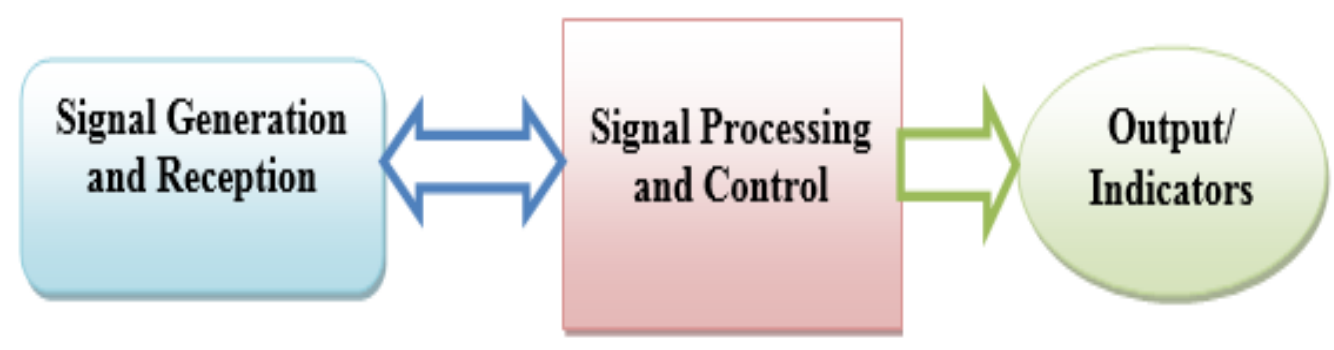

Figure 1. Phase structure of the system

Figure.1 represents the graphical structure of the system. It can be categorized as below,

1. Signal Generation and Reception

2. Signal Processing and Control

3. Output / Indicators

\subsubsection{Signal Generation and Reception}

As the name suggests, this phase is responsible for generation and the reception of the signal which is responsible to detect an object. We can say that this phase is based on the functionality of the sensors. Sensor is a device which converts one form of the energy into another. It is a subset of transducer. It senses the change in the environment depending upon the type of the sensor used. This phase generates a 
typical pattern of the signal which travels all the way and reflects back from the object. Sometimes this module has only receiver which receives the data from surrounding. It can be consisting of a single sensor or an array of the sensors. This sensor can be physical sensors which actually give back the physical data from the surrounding or it can be a pattern of the specific signals which provides us nonphysical information. We have different types of sensors which can fit in our requirement.

\section{a. Optical/visual sensors:}

They give us real time information about the environment. For example the camera devices [14]. They tend to give us accurate results at the cost of the data consumed. We have different kinds of devices/sensors which are based of this category. The typical camera sensors need an exposure to the light for the better operation. For the dark, we can use information. There are some other devices which provide a direct control. It means you can perform different tasks using the single sensor, like heat sensing, movement detection, color detection.

\section{Advantages:}

- They provide a real time operation to the user

- They provide an exact information about the environment at the cost of information

- A single sensor can perform multiple tasks like object recognition, detection.

- They are very easy to install and easy to use.

- Can be controlled using any simple processing board.

\section{Disadvantages:}

- They carry a lot of information which sometimes include unnecessary components

- It fails into the dark. The insufficient exposure to the light may lead to the errors in the reading 
- Infrared camera can work in dark, but again the size of information is a problem

\section{b. Radio Detection and Ranging(RADAR):}

RADAR is an object detection technique. As the name suggests, it uses the radio waves for the operation [15]. By using RADAR, we can get an estimated value of velocity, position and inclination of the object. The transmitter emits radio waves towards the object, whereas the receiver receives the reflected waves. Then the signal is conditioned and processed to extract the prime factors or the data. They are highly accurate, and consume a lot of data. RADAR supports different bands of frequencies depending on the application and type of RADAR used. We have Pulsed Doppler RADAR which is used in tracking of a moving obstacle to determine the range. The Interferometry RADAR is used in healthcare. Continuous Wave RADARs which provide a good protection from the interference. Depending on the application, we can select RADAR which will provides us a real time operation with the system.

\section{Advantages:}

- It can give information about the speed, position, range and inclination of an object.

- They are highly accurate and precise.

- They can work independent of light.

\section{Disadvantages:}

- It works on very high frequency which consumes a lot of data.

- It requires special hardware for signal conversion and conditioning which increase the area of the system.

- It cannot distinguish between the object which are closer to each other.

- Interference is a major problem with RADARs.

- It fails to detect objects which are covered by conductor area. 


\section{c. Light Detection and Ranging (LIDAR):}

As the name suggests, it works on the optical waves. The functioning of LIDAR is same as that of the RADAR. Sometimes it is also called Light-RADAR. It measures the distance with the help of light [16]. It illuminates a beam of LASER (Light Amplification by Stimulated Emission of Radiation) on a target. It works in three spectrums like ultraviolet, visual and infrared. They are highly direction and can map exact information about the object.

\section{Advantages:}

- They can detect a wide range of objects, like conducting, non-conducting materials.

- Its highly directional nature makes it more precise. It has a very high resolution.

- They are used for the terrestrial as well as air borne purposes

- They are very useful in topological mapping

- Highly intense and coherent

- Autonomous vehicles use LIDAR for obstacle avoidance.

- It is very safe and provides a cost effective application in high traffic areas.

\section{Disadvantages:}

- It carries a lot of information which is sometimes unwanted

- It is costly to implement the LIDAR system for very small area/

- LIDAR does not give accurate reading about the slopes.

- It is difficult to map edges using the LIDAR 


\section{d. Acoustic Sensors:}

These types of sensors work on acoustic waves [18]. They transmit the wave into the environment, and receive the reflected wave which is to be processed further. They generally follow a detection mechanism where the properties of transmitted wave are changed because of some factors in environment. Then by comparing the original signal with the received signal, one can detect the factor that affects the system. Typical use of ultrasonic sensor is to detect a range of an obstacle from the sensor. When a signal is transmitted, its amplitude and frequency may get changed because of the object present in the path. From the calculations, we can find out the distance. The advantages and disadvantages of this system can be given as below [20].

\section{Advantages:}

- They are independent of the reflectivity of the surface. They can detect object with the plain surface and rough surface as well.

- They can be used in dark for object detection

- They are very cheap and small in size, easy to install

- They carry very less data as compared to other systems.

- They provide an excellent refresh rate for the detection.

- They are good at motion sensing and distance measurement.

\section{Disadvantages:}

- They have a minimum sensing distance

- They can easily get interfered by change in temperature, humidity etc.

- The plane of the surface to be detected restricts the capacity of detection as the smooth surface has lesser critical angle even if they are good reflector for sound energy.

- Shape of the surface may scatter the waves away from the receiver. 
The comparison between all these sensors can be shown as below,

Table 1. Comparison of different sensors

\begin{tabular}{|c|c|c|c|c|}
\hline Attribute & Visual/Optical & RADAR & LIDAR & Acoustic \\
\hline Size & Small & Small & Small & Small \\
\hline Accuracy & Excellent & Excellent & Excellent & Good \\
\hline Resolution & Excellent & Excellent & Excellent & Good \\
\hline Cost & Moderate & Moderate & Poor & Good \\
\hline Weight factor & Moderate & Low & Moderate & Low \\
\hline $\begin{array}{c}\text { Hardware for } \\
\text { Processing }\end{array}$ & Moderate & More & More & Less \\
\hline $\begin{array}{c}\text { Data } \\
\text { consumed }\end{array}$ & High & High & Very High & Low \\
\hline Applications & $\begin{array}{c}\text { Object } \\
\text { detection, } \\
\text { color, shape } \\
\text { recognition }\end{array}$ & $\begin{array}{c}\text { Range } \\
\text { detection, } \\
\text { object detection }\end{array}$ & $\begin{array}{c}\text { Topological } \\
\text { mapping, } \\
\text { obstacle } \\
\text { detection }\end{array}$ & $\begin{array}{c}\text { Range } \\
\text { detection, } \\
\text { obstacle } \\
\text { detection }\end{array}$ \\
\hline Disadvantages & $\begin{array}{l}\text { Cannot work } \\
\text { in dark }\end{array}$ & $\begin{array}{c}\text { Electromagnetic } \\
\text { Interference }\end{array}$ & $\begin{array}{c}\text { May fail on } \\
\text { slopes and } \\
\text { edges }\end{array}$ & $\begin{array}{c}\text { Less } \\
\text { accurate }\end{array}$ \\
\hline
\end{tabular}

\subsubsection{Signal Processing and Sensor Control}

When the signal is transmitted and received via sensor, one must be aware of the control of the sensors and the process to be followed after receiving the signal. Sensors are the devices which convert physical data into the electrical signal. In order to ensure the sequential operation, one must consider the flow of operation. Before we set up 
our sensor, we should ensure the connection of sensors with the control board. Before which follows a certain flow. In our case, sensors are initialized to send and receive the data. Once the data is received, the next process is done by using the controller or processor. FPGA is also used. We have discussed few most widely used ways for signal processing.

\section{a. Microcontrollers/Microprocessors:}

They are especially designed for the embedded application. Microprocessors are the chips which have a processing unit and accepts a digital data [17]. They do not have on-chip memory and timing control. Most of the times, for embedded systems like household application, microprocessors are used. Microcontroller is a minicomputer where all the things are tight together in a single packet. It has a program memory, data memory, input-output ports and mainly a processing unit. This device can be coded for certain applications. As we know, it gives us a flexible operation, it can be modified easily if there is any change in hardware. Different chips provide different sizes for memory, processing unit and number of input-outputs. The typical input devices are sensors which extract data from environment. The controller is programmed to process the incoming which can be stored at the memory locations present. Depending on the type of sensor, one must select the appropriate chip which is capable of processing such large volume data.

\section{Advantages:}

- They are very flexible because of their programmable nature. We can program them several times without any trouble.

- They are very small in size and also are light in weight.

- They are very cheap in cost.

- They are widely used in embedded applications.

- They are available in different specifications for different applications.

- They also provide data communication. 


\section{Disadvantages:}

- They only work on digital data. So we must use an appropriate device which converts the input data to digital data.

- For the complex operations they fail to provide the flexibility.

- The large data processing can be complex because of the limitations of the chip.

\section{b. Field Programmable Gate Array(FPGA):}

As the name suggests, this device contains large number of logic blocks which are allowed to be connected with each other [21]. This programming is done by Hardware Description Language. It is same as we are connecting very large number of logic gates for the required configuration. Most of the FPGA also contain memory elements which are built using D flip-flops. In short, an FPGA is combination of large number of logic blocks and memory elements in order to carry out complex computation. They have dedicated buses for the I/O data transfer. Some FPGA contain onboard Analogto-Digital and Digital-to-Analog converters on board. They also support mixed signal processing.

\section{Advantages:}

- Because of the parallel architecture, they are very fast as compared to the Digital signal processors.

- The I/O pins can be easily programmed which improves the response time and functionality of the system.

- FPGA ensures a parallel execution irrespective because of the dedicated hardware to perform each tasks and which improves the reliability of the system.

- FPGAs support a non-recurring cost with a good life span.

- They are flexible to the modification and hence provides long-term maintenance.

- They are most widely used in communication, digital signal processing, built-inself-test equipment, 


\section{Disadvantages:}

- They have very large number of logic blocks which are not used in all the applications. As compared to other circuits, it consumes a lot of area.

- Because of the large number of blocks and parallel operation, they consume a lot of power.

\section{c. Application Specific Integrated circuit (ASIC):}

This a dedicated circuit which is manufactured to achieve specific goals. Unlike the general purpose devices, ASIC contains the specific hardware based on the application [22]. They follow the standard cell design which uses gate array along with a full custom design. Modern ASIC may have processor, memory elements and the necessary I/O controls which makes it a System-On-Chip. They are very efficient in high volume operations than FPGAs. Like FPGA, ASIC can be configured by HDL.

\section{Advantages:}

- It supports full custom design approach which allows to manufacture the device as per the designers approach.

- For high volume applications, the unit cost is lower.

- They provide much faster operation because of the full custom approach and gate array.

- DFT can be accommodated in ASIC

- Because of the dedicated specific hardware, it consumes less power than FPGA

\section{Disadvantages:}

- ASIC tools are very expensive, which increases cost for low data volume systems.

- Design to manufacture and signal integrity are major issues with ASIC. 


\subsubsection{Output/Indicators}

Indicators represent specific conditions depending upon their input. In our system, indicators are of great use in order to put all the conditions before the user. It is very difficult to put the environment in front a blind person.

\section{a. Visual Devices:}

Visual devices are nothing but the electronic visual displays which are often used to show images or video. For general purpose navigation system, the visual display can be the perfect type of indicator which is capable of providing all the information about the surrounding. It can give us information about the position, orientation and motion of multiple objects at a time.

For a blind person, it is completely impossible to use such indicators because they are not conveying any information about the obstacles to the user. However, this type of sensors can be used as a reference or a template so that in future, some other indicators can be implemented with respect to the visual devices.

\section{b. Audio Devices:}

Audio device can be of great use for the blind people, as they can audible for a person who cannot see. We can apprise user by giving some information through audio devices. We can also instruct user about the necessary actions to take depending on the surrounding.

However there are some disadvantages of using an audio device. As we know that audio devices can guide a person to navigate, the accuracy is the major issue. For a blind person, it is very difficult to imagine the situation from the given instructions. For example if the object is at left, then the person cannot guess how much left or how high is that object located which makes it risky to use. Also the interference is another issue. Because of the noise, user may not be able to listen to the instructions given. 


\section{c. LEDs:}

LEDs are Light Emitting Diodes which are very simple in structure. LED is about the two possibilities. Either there is an obstacle, or there is no any obstacle. For a visually impaired person, it is not possible to refer LEDs as indicators. But for the reference, we can create a grid map of LEDs which can portray an approximate safe area for the user.

\section{d. Image:}

Images are the most popular way to represent a map of the objects. Like we see in different maps, different objects are located at different places and from which we can find the route to reach to our destination. For a visually impaired person, we can incorporate the same mapping in our system.

But as it is similar to the visual display, user cannot access data from this type of indicators. Hence we need to think about some possible ways to let user know about the obstacles. Body sensing can be a great way to achieve this.

\section{e. Vibrators:}

Vibrator is a mechanical device which operates on electrical signals. It vibrates when we connect the pins of the vibrator to voltage supply. For a blind person, it is simple to sense vibrations over his body. We can implement an array of sensors over the body on different locations. But sometimes we also have to consider about the problems and frustrations the user is facing. It may be annoying sometimes to have sensors all over the body which keep on vibrating. So we must look forward for a new type of sensor or a combination of all these sensor which will make the system efficient. 


\section{CHAPTER III}

\section{PREVIOUS WORK AND LITERATURE REVIEW}

As we have discussed in Chapter I, we studied the basic need of this project. The purpose behind the project and all the possible ways to reach to our goal. Now before we start to work on our system, we must know about the previous literature and work that has been done in the same area. In today's cutting edge technology, many inventions are taking place. When any manufacturer brings his new invention into the market, after some time, another manufacturer comes up with a new invention. But this time he makes sure that his product covers all the flaws which are left in the earlier design. This approach is improving reliability and efficiency of the system. As technology is improving, one must consider the modern ways to work with. This is why, before moving to the implementation, we are going to study the existing technologies which follow the same methodology which we are going to use.

\subsection{Sensor-Cane System:}

This is one of the most widely used system for obstacle detection. We often find visually impaired people carrying a cane. This system is a bit modification over the traditional system. It is an advanced augmented white cane which is capable of detecting obstacles and providing a distance feedback to the user [7]. It consists of three sensors which are directed to the three different directions. The system is very simple and provides user friendly operation.

\section{a. Design and Operation:}

The design of the system is as shown in Figure 2, given below,

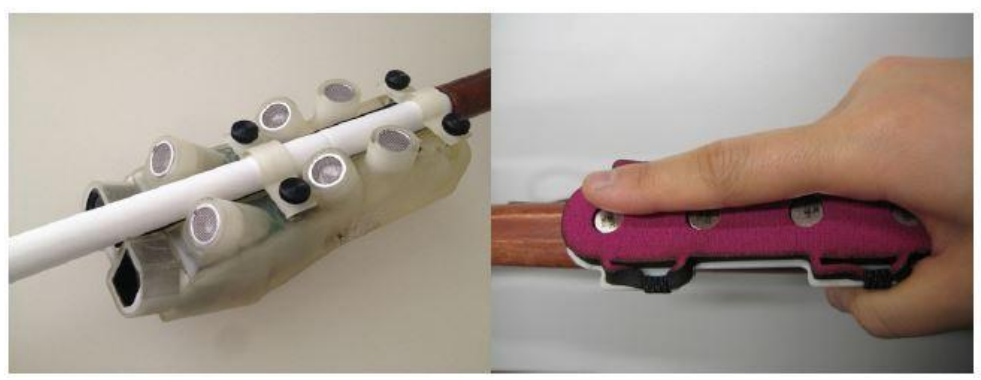

Figure 2. Instrumented cane and vibration handle [7] 
As we discussed earlier, three ultrasonic sensors have been placed on the cane. Each sensor is responsible for different direction. It also contains an infrared sensor which is placed at the bottom. The handle of the cane is consisting of 4 vibration motors. Each of the motor is associated with a sensor. This can be explained using the figure given below,

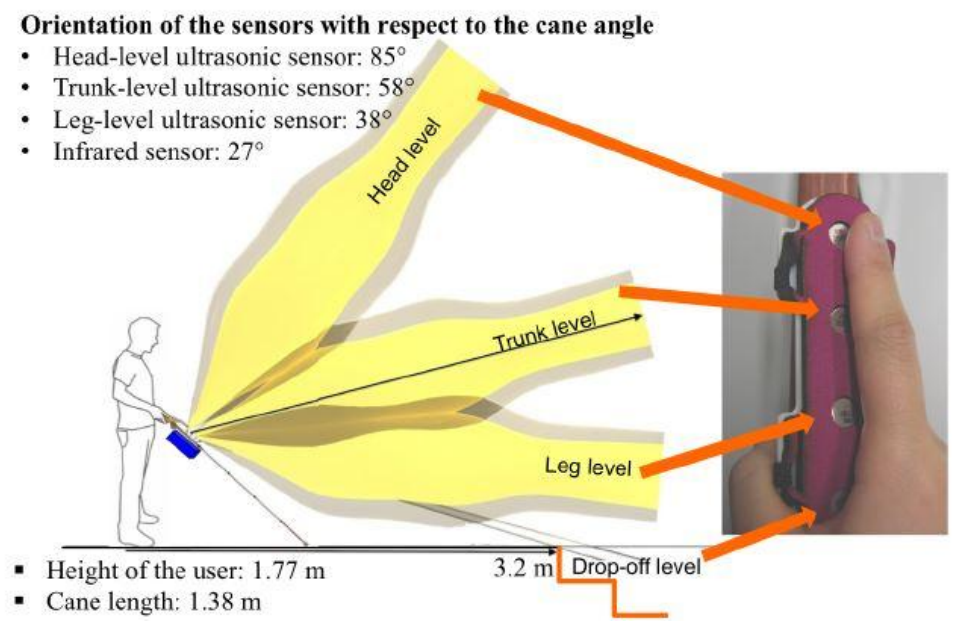

Figure 3. Sensor arrangement and specifications [7]

The sensors are divided into four regions. As we can see in Figure 3, upper sensor is head level, middle sensor is trunk level and bottom sensor is for leg level. Also infrared sensor is used for drop-off level. Each vibrating motor is associated with the sensor.

Experiments were performed to verify the functionality of the each sensor. It followed two way operation. Firstly with the steady cane, and secondly with the sweeping cane. Each of the experiment gave nearly accurate results. System failed to detect drop-off in number of experiments.

\section{Advantages:}

- System gave nearly accurate results for overhead, front and bottom obstacle detection.

- It uses very minimal hardware system for detection.

- Provides a user friendly operation as system is following a traditional approach. 
- Vibration motors are very efficient to inform the user about the possible obstacle location.

\section{Disadvantages:}

- Traditional approach fails to provide a hands-free operation to the user

- Three level obstacle detection approaches fails when there is an incoming target from different direction.

- System cannot provide information about the obstacles which are left and ride side unless we swipe the cane.

\subsection{Wearable Jacket system:}

In this system, a different methodology has been followed which uses ultrasonic sensors which are mounted on a jacket [1]. System uses SONAR approaching. That is Sound Navigation and Ranging. Improved approach has been followed to improve the detection range and to make system user friendly. We will go further to talk about the system

\section{a. Design of the system}

The design is based on the embedded systems and they have introduced a new term called textile wearable system. This design is capable of classify obstacle depending on their nature. Ultrasonic sensors are implemented over the textile design, which are controlled using a microcontroller board. It mainly supports indoor environment.
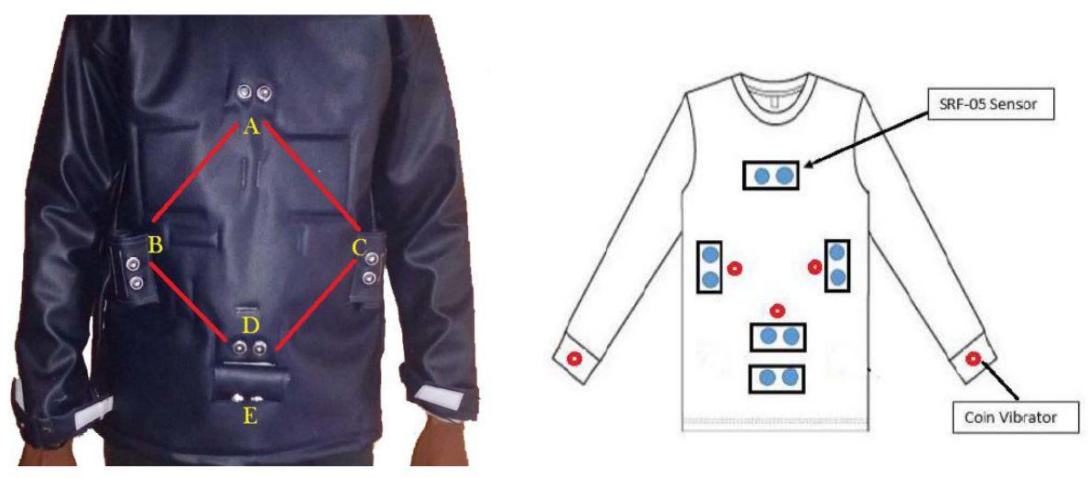

Figure 4. System Architecture [1] 
The system comprises of three phases, sensing module, signal processing module and feedback module. As we can see in Figure 4, five ultrasonic sensors are mounted on the jacket which is responsible for five different directions. Signal processing module is built using microcontroller board. Vibrators are used on the both hands to give feedback to the user. This feedback can help user to divide the region into safe region and risky region.

Sensing module is responsible for the signal transmission and reception. The physical environment is sensed and converted in terms of the electrical signals. Ultrasonic sensors are used. The signal processing module primarily carries out operations like, wait time, range. It uses microcontroller board. When signal is received, the information is processed by the board and then specific signal is sent to the vibration module. They give an indication to the user depending upon the received signal

Sensor placement has been very critical point while designing the system. As shown in Figure 2, sensors A, B, C, D and E are placed on the jacket. Sensors A, B, C and D are focused towards the front path, whereas Sensor E ensures the fall detection. Sensors are inclined to each other in such a way that they provide minimum overlapping. Microcontroller is placed nearer to the all sensors. Batteries are used to provide a power supply to the design. The Netduino- 2 microcontroller board is used in the signal processing module.

\section{b. Operation:}

Basic purpose was to satisfy three criteria. Firstly indoor targets such as edges, corner, plane and open area. Secondly ground level objects like pit, staircase or any other obstacle. Thirdly object which has different shapes and surfaces areas like wall, window, chair etc. Distance of an obstacle from each sensor is calculated using the time of flight of the respective sensor.

Experiments were carried out. User who is wearing this jacket moved around the indoor environment at first. After that he was asked to move on slope, staircases. And at the end, all kinds of objects were included and readings were taken. 


\section{c. Results:}

First experiment gave much better results. It is mainly because of the surface of the objects. Plain surface is a good reflector. Whereas the corners, edges failed to give a reflection. In second experiment, it was nearly accurate because of the sensor limitation whereas in the third experiment, plain surfaces were detected without any error whereas the complex shaped object were causing errors and accuracy was around $88.9 \%$.

\section{d. Advantages :}

- System is portable, can be carried with ease.

- System uses very less hardware consuming less power.

- Systems behavior was very good under plain surfaces.

- Wearable cloth is durable and washable.

\section{e. Disadvantages:}

- System is not completely reliable.

- More number of sensors are needed for ground level operation.

- It fails to cover the objects which are above the height of the sensor position.

- Increasing number of sensors in the system may affect the existing hardware.

- Corner and edges showed very low accurate results.

\subsection{Smart Backpack System:}

This system consists of ZigBee modules which detects the range of objects by processing the received signal. They have also built a grid based map to help user. Ultrasonic sensors are also used for obstacle detection. System is made wearable by using the backpack [4].

\section{a. Design and operation:}

The design of the system is very simple in nature. It is divided into two parts. Tracking part and detection part. ZigBee module detects the range of an obstacle from the user. Mobile computer processes the data to estimate the position. 


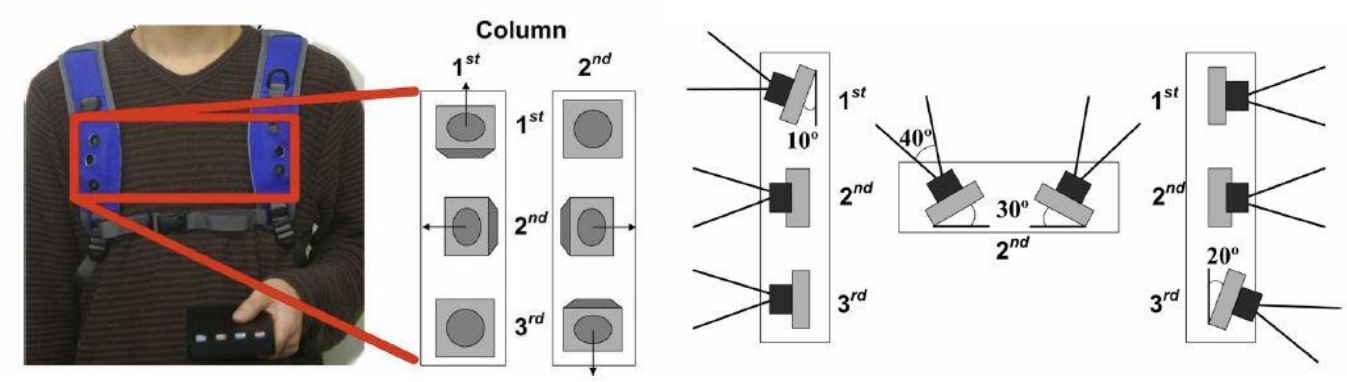

Figure 5. Layout and geometry of ultrasonic sensors [4]

The main goal was to cover the maximum range using the minimum number of sensors. So array of sensors is used. They have implemented two arrays each consisting of three sensors. As you can see in Figure 3, each of the sensor is responsible for different angle. This is to achieve the maximum area by using less number of sensors. The upper sensors are responsible for the upper part and lower sensors cover the ground level operation. Middle sensors cover the left and right hand side obstacles.

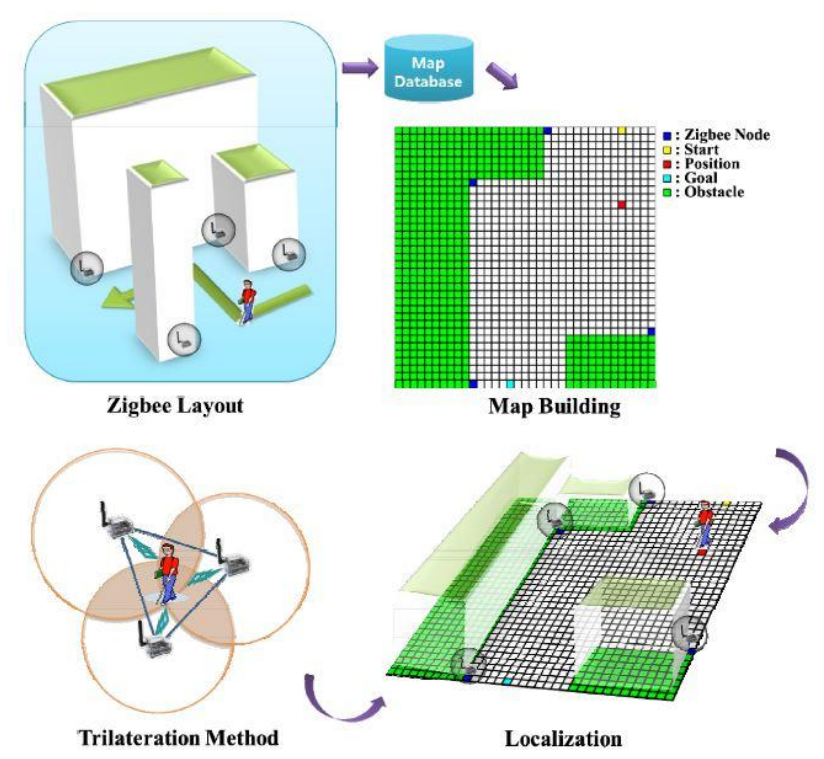

Figure 6. ZigBee modules for position estimation [4]

ZigBee modules are implemented for the position approximation. As you can see in Figure 4, ZigBee modules are placed on the path of the user. ZigBee modules transmit and receive signal. Depending on the signal strength, range is calculated. This 
system is useful in approximating the position in the path. As you can see in Figure 4, ZigBee layout is consisting of modules. All sensors can act parallel to approximate the position of the person.

Using this approximation, we can set up a map which can locate the position of user in a plane. Numbers of experiments were carried out. The accuracy was found to be $95 \%$ and $10 \%$ of the results were affected by interferences.

\section{Advantages:}

- System is wearable and easy to carry along with user.

- System uses very less hardware, hence consumes very power

- System is $95 \%$ accurate, hence good for indoor communication.

\section{Disadvantages:}

- As the ZigBee modules are fixed for particular location, the outdoor operation of the system

- Wireless interference is a major issue

- Visually impaired person cannot predict the nature of the object.

- Lack of mapping technique in blind person's point of view.

\subsection{Wearable Audio Assistance:}

This project is about the system, where a wearable device is used for indoor obstacle detection [6]. The audio assistance is used to assist the blind people. Additionally a camera sensor is used for the guidance. The design and operation of the system is as follows.

\section{a. Design and operation:}

System is consisting of two ultrasonic sensors and a camera sensor. These sensors are mounted on the glasses. The camera is places in the middle of the glasses whereas the sensors are placed at the edges. Audio device is used which instructs user upon the detection of obstacle. The system looks like as given below. 


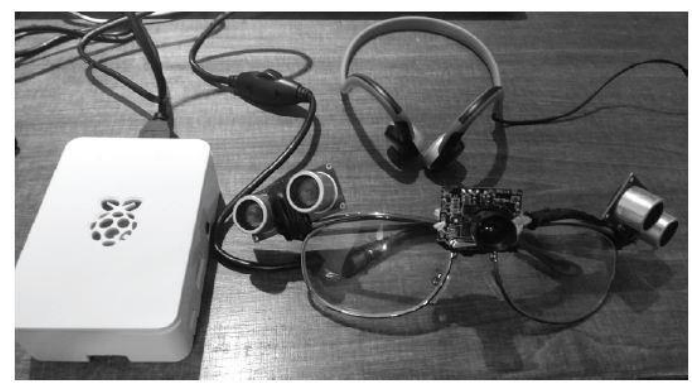

Figure 7. System structure [6]

A microcontroller board controls the ultrasonic sensor. They have also used one gyroscope, magnetometer and one accelerometer. The software module takes care of the computer vision. It is used to recognize different obstacles using a computer. The ultrasonic module takes care of the functioning of the sensors. Computer vision uses image processing techniques to get a simpler version of the obstacle map.

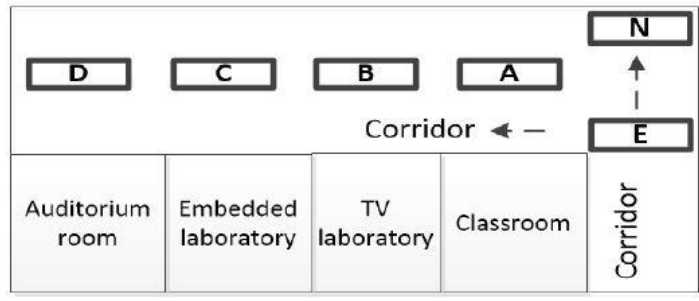

Figure 8. Free mode navigation [6]

Figure 8 represents the experimental set up for the system. Number of markers is placed through a corridor. Each marker is associated with specific sound instruction. Whenever user sees markers, corresponding instruction is played through the audio device. An average of $94.92 \%$ success for markers detection and $98.33 \%$ for obstacle perception.

\section{Advantages:}

- System provides a portable and wireless operation.

- Camera sensor increases the accuracy of the system.

\section{Disadvantages:}

- As camera sensors are used, it is not helpful for the user who is visually impaired.

- Also camera consumes a high volume of data.

- It may fail in the dark because of the lack of the exposure. 
- Ultrasonic sensors are not enough to cover all of the information about the possible obstacles. They can only give an idea about the left or right side obstacles.

- The information about the range is not provided.

\subsection{Human Fall Detection:}

In this project, a system is designed which is capable of detecting the activity of a person in a room. Areas where we find it difficult to place cameras to keep an eye on a person. This system can be implemented. This system does not provide any aid for the blind navigation but it can help us with positioning and activity monitoring. Let us discuss about the design and methodologies followed in this project.

\section{a. Design and operation:}

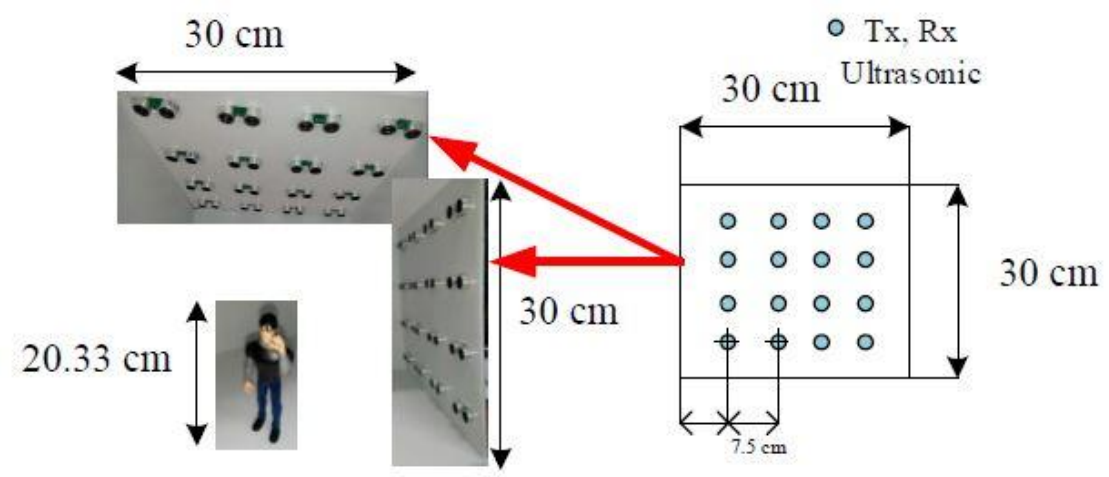

Figure 9. Structure of the system [5]

Figure 9, shows us the actual structure of the system. We can see that in a room, an array of ultrasonic sensor is placed. Each sensor undergoes through transmit and receive operation.

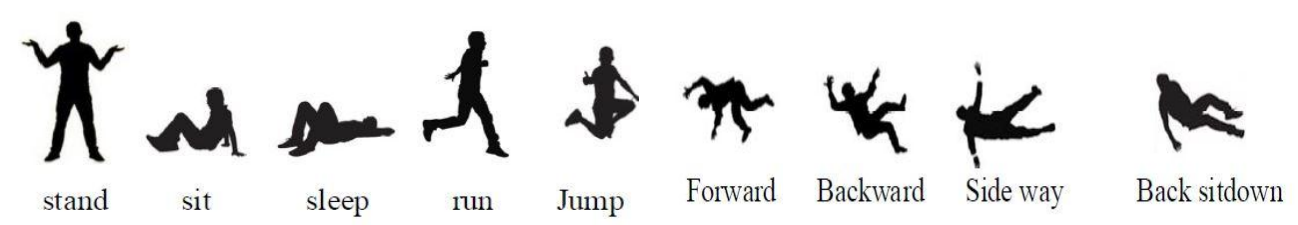

Figure 10. Different activities for detection [5]

Figure 10 shows us the different activities which can be performed by the person under the observation. Two arrays of ultrasonic sensor detect the activity of the 
person. One array is placed at the top of the room whereas the other array is placed on the wall. Readings were taken.

Ultras onic Sensor Array
\begin{tabular}{|c|c|c|c|c|}
\hline 1 & 1 & 2 & 3 & 4 \\
\hline & 0 & 0 & 0 & 0 \\
\hline 2 & 0 & 16.5 & 16.5 & 0 \\
\hline 3 & 0 & 0 & 0 & 0 \\
\hline 4 & 0 & 0 & 0 & 0 \\
\hline
\end{tabular}

Standing top
Ultras onic Sensor Array

\begin{tabular}{|c|c|c|c|c|}
\hline 13 & \multicolumn{2}{|c|}{1} & 2 & \multicolumn{3}{c|}{3} & \multicolumn{1}{c|}{4} \\
\cline { 2 - 6 } & 0 & 0 & 0 & 0 \\
\cline { 2 - 6 } & 0 & 18 & 18 & 0 \\
\cline { 2 - 6 } & 0 & 18 & 18 & 0 \\
\hline 4 & 0 & 18 & 18 & 0 \\
\hline
\end{tabular}

Standing side

Figure 11. Mapping of the activity [5]

From Figure 11, it is clear that person is right below sensor 2 and 3. Also from side sensors. We can verify that person is standing at the respective position. The accuracy of the system was found to be $92 \%$.

\section{Advantages:}

- The more number of sensors give more information about the physical state of the person

- Mapping of the sensors give us a three dimensional idea about the position.

- It is simple in nature, performs simple operation of range measurement.

\section{Disadvantages:}

- System does not provide any aid in navigation.

- Area for the measurements is limited to a room.

- System is not wearable or portable.

\subsection{Grayscale Mapping:}

This paper tells us about the new technique that has been implemented to map the obstacles around the user [2]. We are mainly focusing about the mapping part of the paper, which is helpful in our system in the future. 
a)
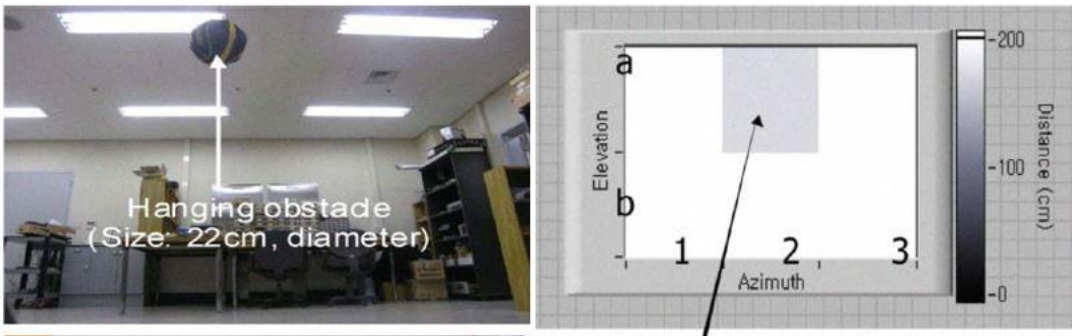

b)
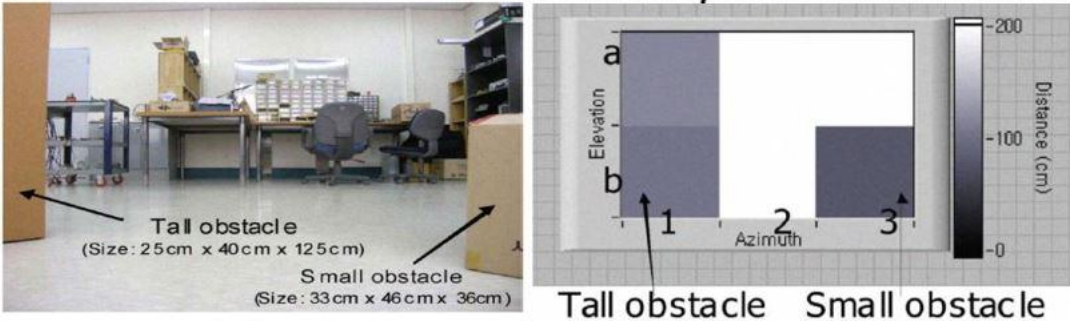

Figure 12. Obstacle distribution and mapping [2]

In Figure 12, we can see the mapping of obstacles. They have used the grey scale imaging. The plane is divided into six parts. Each reading is given by an ultrasonic sensor. Depending upon the distance given by the sensor, objects are mapped. Closer is the object, darker is the value. Whereas the white block represents the object which are far from the user.

\section{Advantages:}

- Mapping techniques is very useful and can be used in array format of vibrational motors.

- It can be considered as a three dimensional mapping as the closest and farthest can be easily separated.

- This method gives an exact idea about the safe zone to be followed by a person.

\section{Disadvantages:}

- This type of mapping alone cannot help a blind person for navigation as there is no any way to communicate between this mapping and the user.

- System uses only 6 sensors which sometimes may not give any idea about the pit in front of the user. 


\section{CHAPTER IV PROPOSED SYSTEM}

So far we have studied all the methodologies to implement the system for blind navigation. Also we have studied the previously working methods/systems which are used and popular. Now depending upon this study we now have to implement our design, which is a third phase of this project. From all the systems we have studied so far, we should consider all the possibly ways which will help us to achieve our goal to make system portable, wearable and capable to detect obstacles. Also mapping technique should be simple enough to inform user about the possible obstacles in his way.

Now in the first phase of signal generation and reception, we are going with ultrasonic sensors. They carry very less but important information about the range of the objects. Also they are easy to install and program. In the second phase of the signal processing, we are going with a completely new approach. This approach uses devices which are all in one board. The third phase that is output/indicator is using the grayscale mapping and vibration motors. Also we have implemented a 3D mapping technique which can actually inform us about the projections caused by each object. Let us discuss about the different system phases.

\subsection{Phase Structure of the System}

The system is divided into three phases as we have discussed earlier. The graphical view of the system can be shown using Figure 13. First phase uses ultrasonic sensor array. This sensor array is responsible to transmit and receive signal from the objects in their path. The signal processing is achieved by using NI myRIO1900. This device is manufactured by National Instruments. The purpose behind this device is to provide a flexible and multi circuit operation to the user. It also decreases the hardware required to perform the multiple tasks. It is controlled using LabVIEW. The 
output is generated using grey scale map, and also a three dimensional map. These maps are generated using LabVIEW and MATLAB.

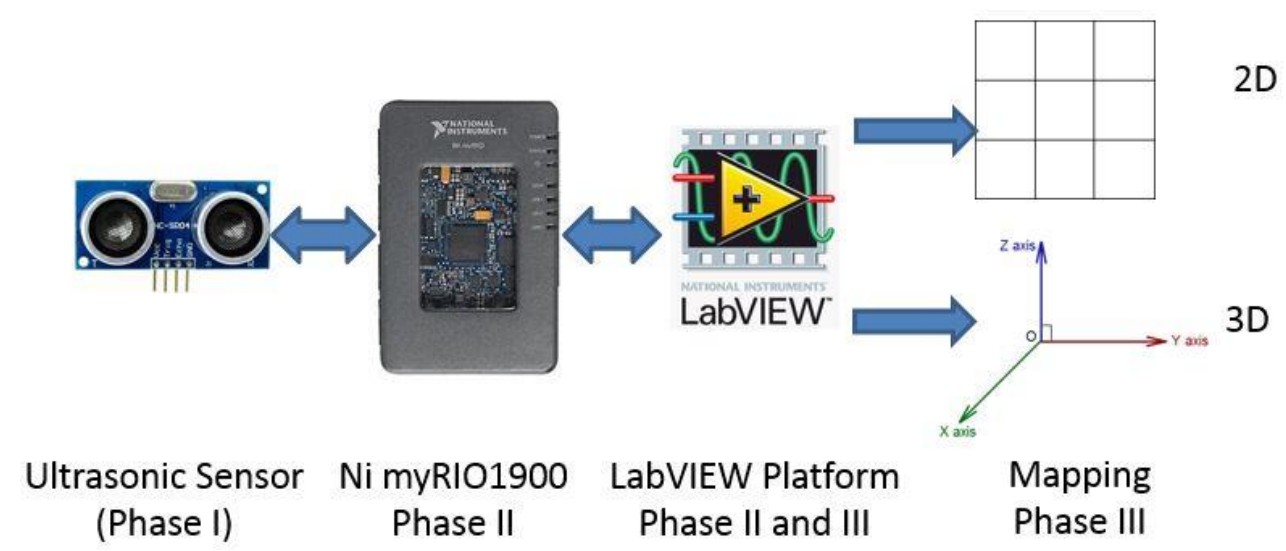

Figure 13. Proposed system architecture

Figure 13 can help us to realize the actual flow of the operation of the system. LabVIEW is the main component in this system as it processes the signal and produce output. Also NI myRIO 1900(here after will be referred as myRIO) interfaces sensors and controls transmit and receive operation. Depending upon the received signal, LabVIEW generates the output. Now we will discuss each phase in detail.

\subsubsection{Ultrasonic Sensor}

We have previously discussed about the basic definition of the acoustic sensors. Ultrasonic sensors are very popular for range detection. This sensing technique is a SONAR technique which can also be seen in some animals like dolphins and bats. We have used HC-SR04 by Cytron Technologies [23]. This sensor offers very high accuracy for range detection. Also the stability of the readings is found to be high. They are capable of finding hard as well as soft materials.

\section{a. Design:}

The HC-SR04 module is a four pin sensor. Two pins ensure the power supply whereas one pins is for transmission and one for echo reception. It works on a $5 \mathrm{~V}$ voltage supply. Figure 14, given below, shows us the structure of the sensor. We have both transmitter and receiver on the same chip. When we give a high pulse to the 
transmit pin, it triggers a chirp signal, which get reflected back from the object and received by the echo pin.

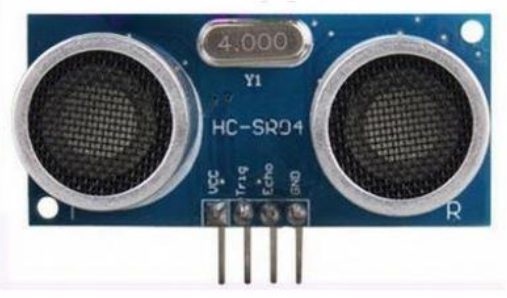

Figure 14. Ultrasonic sensor HC-SR04 [23]

\section{b. Specifications:}

- It works on a power supply of 5V DC.

- Frequency of operation is $40 \mathrm{KHz}$.

- Typical Quiescent current is less than 2mA.

- Typical operating current is $15 \mathrm{~mA}$.

- Range is from $2 \mathrm{~cm}$ to $400 \mathrm{~cm}$.

- The typical measuring angle is $30^{\circ}$ where it can deviate depending upon the surface.

- Maximum effectual angle is $15^{0}$.

- It generates a trigger pulse of $10 \mu \mathrm{S}$.

\section{c. Performance and Operation:}

Practical test performance of the sensor system is pretty good within the angular range of $30^{\circ}$. Figure 15 shows us the typical performance of the sensor.

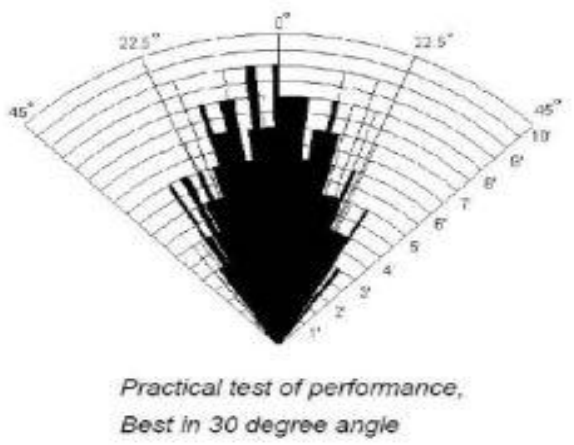

Figure 15. Testing of the performance for angular range [23] 
Connect the power supply to the sensor. When transmitting pin receives a $5 \mathrm{~V}$ supply for at least $10 \mu \mathrm{S}$, it gives a burst of eight cycles at $40 \mathrm{KHz}$. When this wave reflects back from the object, echo pin shows a high value that is $5 \mathrm{~V}$ for a time depending upon the distance it travelled. Time for travel is the time taken by pulse to reach to the object and reflect back to reach receiver. If ' $d$ ' is the distance, and ' $t$ ' is the time of flight, then the distance can be calculated as follows,

$$
d=\left(\frac{t}{2}\right) * v
$$

Where $v$ is the velocity of sound in air at room temperature. Typical value of $v$ is $340 \mathrm{~m} / \mathrm{S}$. The distance is calculated in meters.

The triggering operation can be explained using the Figure 16, given below. The width of the received pulse decides the distance of an object from the sensor. Larger is the distance, larger is the width of the pulse.

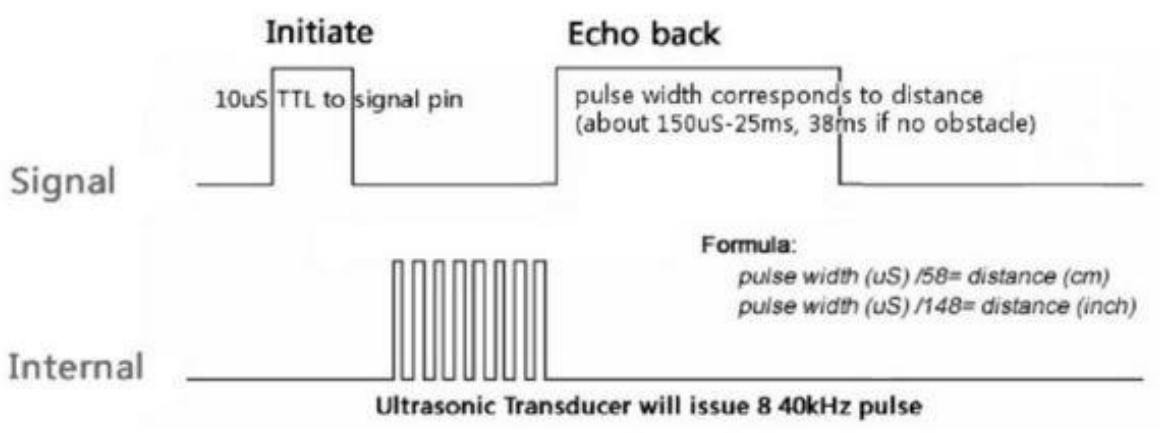

Figure 16. Trigger and echo pulse [23]

Specifications and limitations of the sensor can be explained using the table given below,

Table 2. Specifications and limitations of HC-SR04

\begin{tabular}{|l|c|c|c|}
\hline \multicolumn{1}{|c|}{ Parameter } & Min & Typ & Max \\
\hline Operating Voltage(V) & 4.5 & 5 & 5.5 \\
\hline Quiescent Current(mA) & 1.5 & 2 & 2.5 \\
\hline Working Current(mA) & 10 & 15 & 20 \\
\hline Operating Frequency(KHz) & - & 40 & - \\
\hline
\end{tabular}




\subsubsection{NI myRIO1900}

NI myRIO 1900 is a Reconfigurable - Input - Output (RIO) device, manufactured by National Instruments [9]. It provides an all in one operation to the user. Also widely used to design control, mechanical and robotics system. It is a multiple design concept device. It is based on previously used architecture. LabVIEW platform is used for the myRIO again this time. It contains a processor, FPGA and bunch of I/O ports which are completely programmable through LabVIEW. It uses Xilinx Zynq technology which is all programmable and provides a System-On-Chip module.

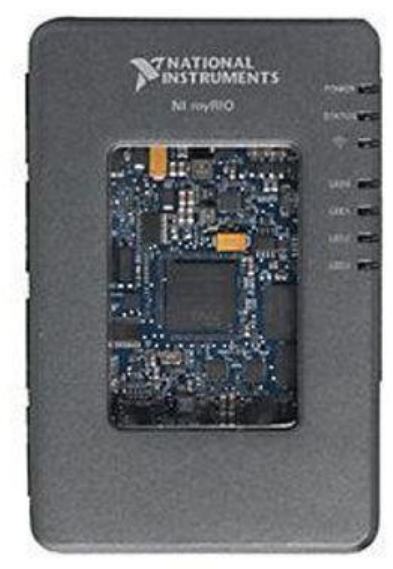

Figure 17. NI myRIO 1900 [9]

The specifications of the RIO can be given as below,

\section{a. Specifications:}

- It needs a supply voltage of 6-16V.

- It provides 40pin digital input-output lines which operates on 3.3V

- It also provides 10 analog input channels which internally are connected to a single analog to digital converter.

- It has 6 analog output channels which have a dedicated digital to analog converter.

- Audio input and output ports are also connected to ADC and DAC and which can be connected to headphones are microphones directly. 
- Accelerometer present on the board samples and provides a continuous data about the axes and keeps registers updated.

- It also supports serial communication. It has one UART transmit and one UART receive line. These lines are identical in nature with the DIO lines.

- USB port on the board allows adding a flash drive or camera to it.

- It also has an IEEE802.11 b,g,n wireless module on board.

- Push button is for error debugging which is an additional feature.

The architecture of the NI myRIO 1900 can be shown as in Figure 18.

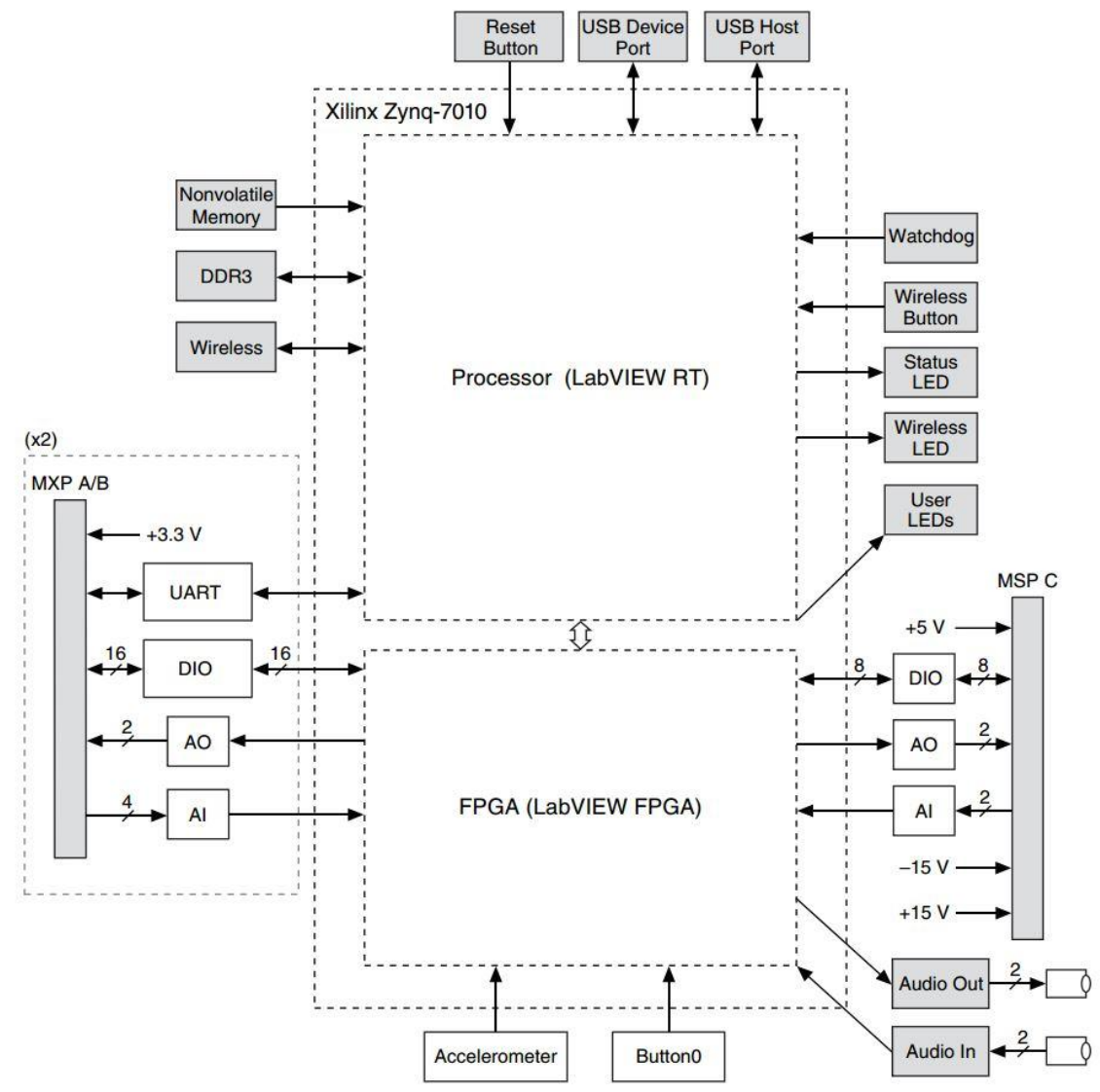

Figure 18. MyRIO architecture [9]

Xilinx7010 FPGA board provides multiple features to the system. It has dual core ARM Cortex9 processor which provides a high speed operation. It provides a non-volatile memory of $256 \mathrm{MB}$ and DDR3 of 512MB. 
NI myRIO provides a wide range of applications in all the major fields. Digital signal processing, mechanical as well as control systems, DC drive etc. In our project, NI my RIO provides a flexible operation. Wi-Fi modules make our system portable as well as wireless. RIO is very light in weight and can be carried along. It consumes very less space. Power consumption can be a problem for smaller systems. It offers an on-state power consumption of $14 \mathrm{~W}$ and idle consumption of $2.6 \mathrm{~W}$. However if we neglect this power consumption caused by RIO, it can be a better system in future as we can use many of the features present on RIO.

\subsubsection{LabVIEW}

LabVIEW is a platform that connects the myRIO for the processing. LabVIEW is very popular designing tool which provides an ease of access introducing a new way to design. It is nothing but graphical programming language which makes it more user friendly. In our project, LabVIEW is going to perform the following tasks.

\section{a. Configuring I/O ports of myRIO:}

The sensors are connected to myRIO and which can be accessed using the LabVIEW. This is the important part in designing because before writing the code in LabVIEW, we must have access to the I/O pins of the RIO.

\section{b. Processing the received signal:}

When the signal is received, LabVIEW performs a set of operations to detect the range of the object. It also generates the output based on the received signal.

\section{c. Output generation:}

As per the calculations done by processing module of LabVIEW, output is generated in terms of a two dimensional and a three dimensional images using LabVIEW.

\subsubsection{Mapping:}

In the mapping section, we are focusing on grayscale imaging and a $3 \mathrm{D}$ image generation. The grayscale image will be in terms of two dimensional image. Though the image contains three variables, we are going to consider it as a two dimensional 
image as the third variable does not provide an exact information about the actual distance to the user.

\section{a. Two Dimensional Mapping:}

Two dimensional Mapping mainly depends on a grayscale format. Now what is a grayscale image? Answer is that the grayscale image is a combination of black and white color [2]. It is defined by specific values. The black color is set with the ' 0 ' value, whereas the white color is set to ' 255 '. Now from this $0-255$ range, we see 256 combinations of colors [10]. Each of the value represents a grayscale value. In our project, we have used a normalized structure to generate the image. The maximum distance for the measurement is set to be 2 meters. If ' $G$ ' is the grayscale factor and ' $\mathrm{d}$ ' is the measured distance, then the grayscale factor for each measurement can be calculated as below,

$$
G=\left(\frac{d}{2}\right) * 255
$$

The grayscale factor can be tabulated as below,

Table 3. Grayscale Distribution

\begin{tabular}{|c|c|c|}
\hline Grayscale & Distance & Dist. in meters \\
\hline 0 & Closest & $0 \mathrm{~m}$ \\
\hline 255 & Farthest & $2 \mathrm{~m}$ and greater \\
\hline
\end{tabular}

\section{b. Three Dimensional Mapping:}

Three dimensional mapping of the objects detected by the sensors can be representing by the projection given by the each measurement. If we consider the front plane, then each of the objects present in that plane gives us a third dimension in terms of the projections given by the plane onto the user. 


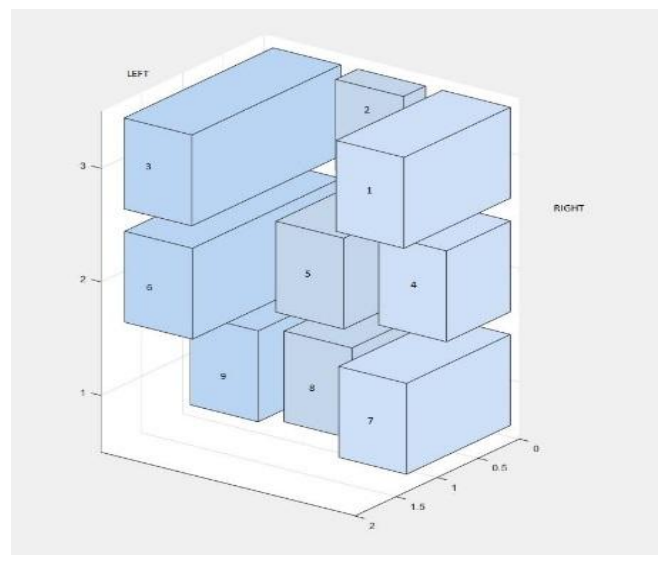

Figure 19. Three dimensional mapping

As we can see in Figure 17, the left hand side of the image has projects which indicated that the object is closer to the user. The center top box indicates that the object is far from that sensor.

These mapping systems can help us in future to map the actual indicators over the body. This can be the baseline for the different techniques that we are going to use in future. 


\section{CHAPTER V}

\section{EXPERIMENTAL WORK}

This is the last stage of our project. So far we have discussed everything about the history of such systems. We implemented the system using an array of ultrasonic sensors, Signal processing can be done using myRIO and LabVIEW. Also we can generate the output using the LabVIEW. The next part to be done is testing of the system. Testing includes few basic experiments at first which will allow us to set the limits for our system. It necessary in order to calibrate our system with respect to the sensors. We should have an idea about the behavior of the system for a single sensor. Depending on which we can modify our system in order to get more readings.

The primary work is to decide the flow of operation. It is necessary for the reliable and accurate operation of the system. A certain sequence of operation should be followed that ensures the flow.

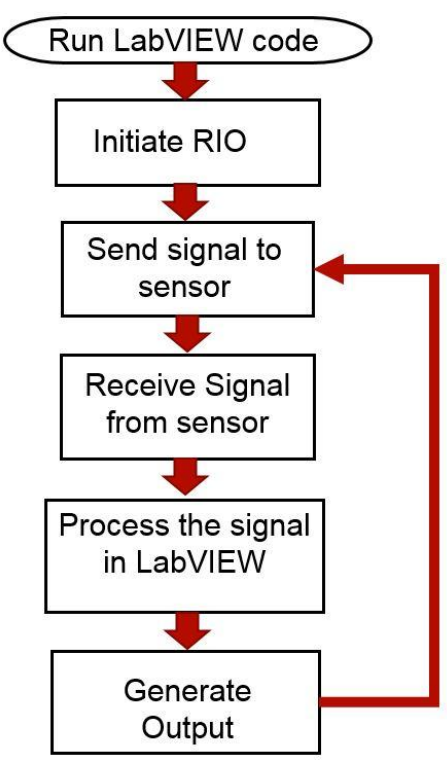

Figure 20. Operational flow

Figure 20 indicates a flowchart for the operation. Initially we establish connections between sensor array and RIO. We then connect the RIO to our system using wireless module. We write the code for our assembly in LabVIEW. Once we are done with the 
coding, we can run the code. That is the very first attempt to start operation. When we run our code, it is deployed on the FPGA present on the RIO. It initializes RIO for the signal transmission and reception. Next step is to transmit the signal from sensor. This is achieved by providing a pulse signal to the transmitter pin of the sensor. Signal travels through air and reflects back from the obstacles. This signal is received by the echo pin of the sensor. This signal is then feed back to the LabVIEW via RIO. This signal is then processed to find out the time of flight of the signal. Pulse width modulation is used to find the time elapsed. Once the time of flight is calculated, distance is calculated using the formula. Depending on the values generated, LabVIEW generates the output in terms of two dimensional grey scale map and a three dimensional projection map. Again system sends signal, receives it, and generates the output which is a continuous loop which can be interrupted using the stop button provided in LabVIEW code. This operation provides a real-time operation of the system to the user. Let us discuss about the experiments and testing of the system for different number of sensors.

\subsection{Single Sensor System:}

A single sensor system consists of only one sensor connected to the RIO. The basic purpose behind this experiment is the calibration. Each sensor in our system is going to give us information about the range of the obstacle and the wide angle of the detection. Single sensor will help us to calculate the accuracy of the system. While calculating range, we must know the minimum distance that can be measured with our system. We also need to know the angular range of the sensor. For example if the object is towards the right hand side, then at what angle obstacle can be detected. While considering the side lobe angle of the sensor, we must know about the vertical lobe angle too. The lobe size increases as we move away from the sensor. The vertical angle is very small in the order of $5^{0}$.

In this experiment, we have connected a sensor to RIO on a plain horizontal surface. An object is moved around the sensor for different positions. The experimental set up can be shown as in figure below. 


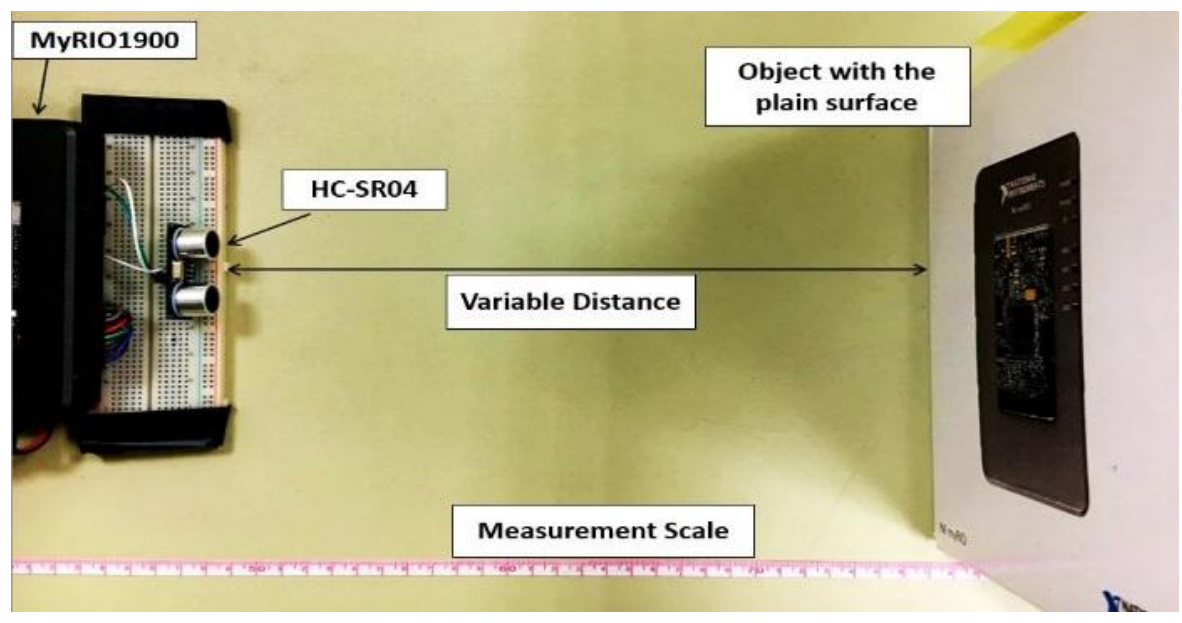

Figure 21. Experimental setup for Single Sensor System

Experimental setup for the system is as shown in the Figure 21. An obstacle with a plain surface is placed in front of sensor. Sensors transmit and receives signal. As we move our obstacle closer or away from the sensor, the distance measurement is also changed. The position of the sensor is fixed whereas the obstacle is movable. Distance is measured using the measurement tape.

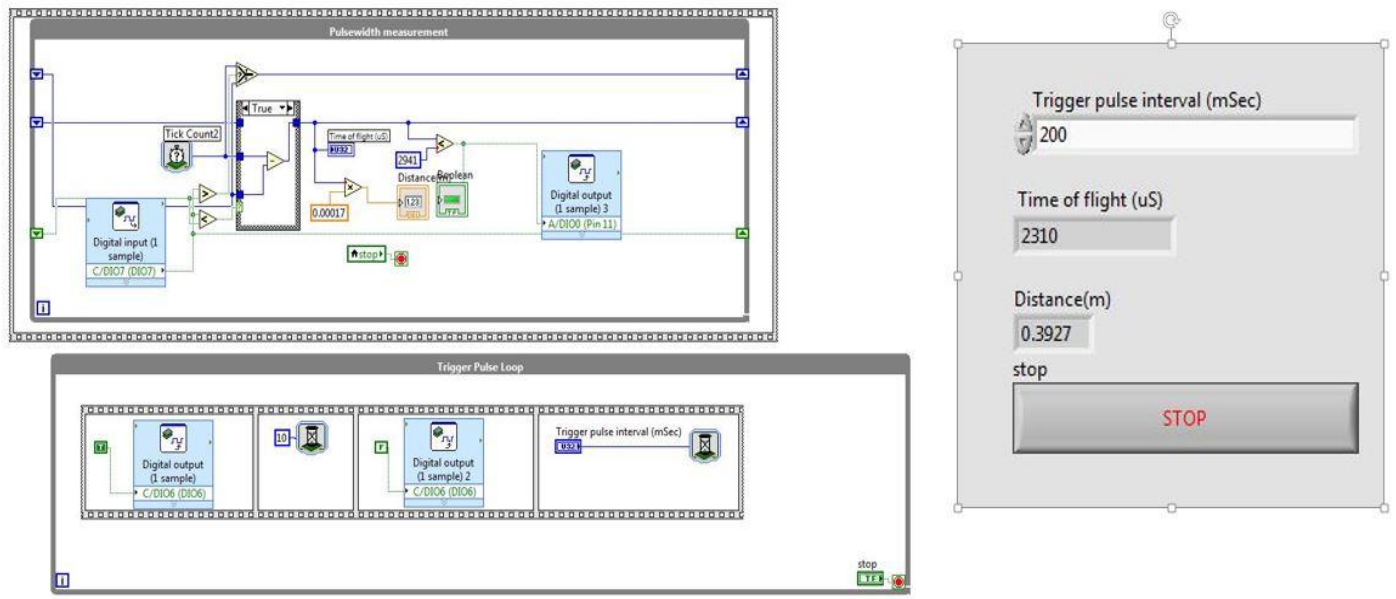

Figure 22. LabVIEW code for Single Sensor

In Figure 22, the sample LabVIEW code for a single sensor is given. Code consists of two parts. Implementation part consists of code for transmission and reception. Graphical part represents inputs and outputs. This code is modified to perform the single sensor operation. 


\subsubsection{Distance measurement:}

Distance measurement is done in terms of number of sets. We have considered 12 sets. We have considered values from $0.1 \mathrm{~m}$ to $1.2 \mathrm{~m}$. We took 10 readings for each of the set. We then calculated an average distance value in each set. Following table represents the comparison of an average distance for each set.

Table 4. Distance Measurement

\begin{tabular}{|c|c|c|}
\hline Set Number & Actual Distance(m) & Measured Distance(m) \\
\hline $\mathbf{1}$ & 0.1 & 0.1002 \\
\hline $\mathbf{2}$ & 0.2 & 0.2014 \\
\hline $\mathbf{3}$ & 0.3 & 0.2988 \\
\hline $\mathbf{4}$ & 0.4 & 0.3942 \\
\hline $\mathbf{5}$ & 0.5 & 0.4969 \\
\hline $\mathbf{6}$ & 0.6 & 0.5969 \\
\hline $\mathbf{7}$ & 0.7 & 0.69294 \\
\hline $\mathbf{8}$ & 0.8 & 0.8018 \\
\hline $\mathbf{9}$ & 0.9 & 0.894 \\
\hline $\mathbf{1 0}$ & 1 & 1.018 \\
\hline $\mathbf{1 1}$ & 1.1 & 1.112 \\
\hline $\mathbf{1 2}$ & 1.2 & 1.191 \\
\hline
\end{tabular}

Table 4 shows us the mapping between actual and measured distance. As we can see the measured distance doesn't differ by a larger value with the actual distance. So we calculated an average error per set.

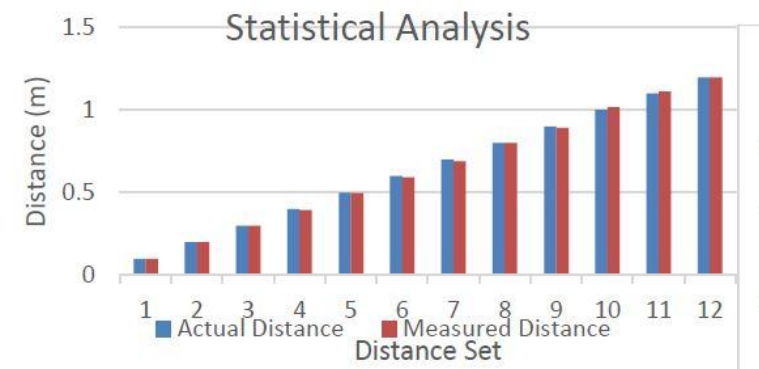

(a)

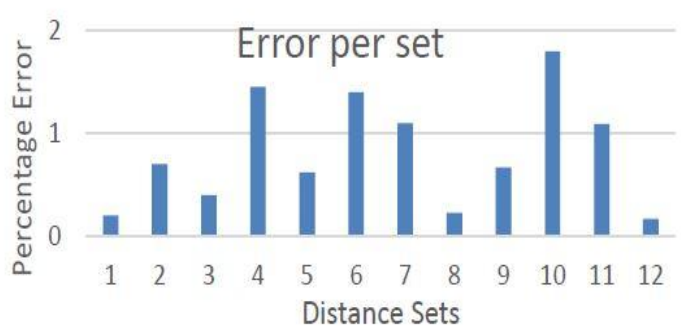

(b)

Figure 23. Percent error per set

The accuracy of the measured distance depends on the average value of distance calculated in each set (Figure 23.a). Hence the average error caused in distance measurement was found to be $0.8 \%$ (Figure 23.b). This value is almost accurate which makes our system more reliable. 


\subsubsection{Angle Measurement:}

The next parameter to be evaluated is the angle of detection. As we have discussed that the sensor gives very small vertical angle for measurement, we will mainly focus on the horizontal angle. This term can be referred as 'detection zone'. In order to do this calculation, we carried out two experiments. In first experiment, we placed an obstacle on the right hand side of the sensor. Similarly we did the experiment for the obstacle at left hand side. The experimental setup can be shown as in figures below.

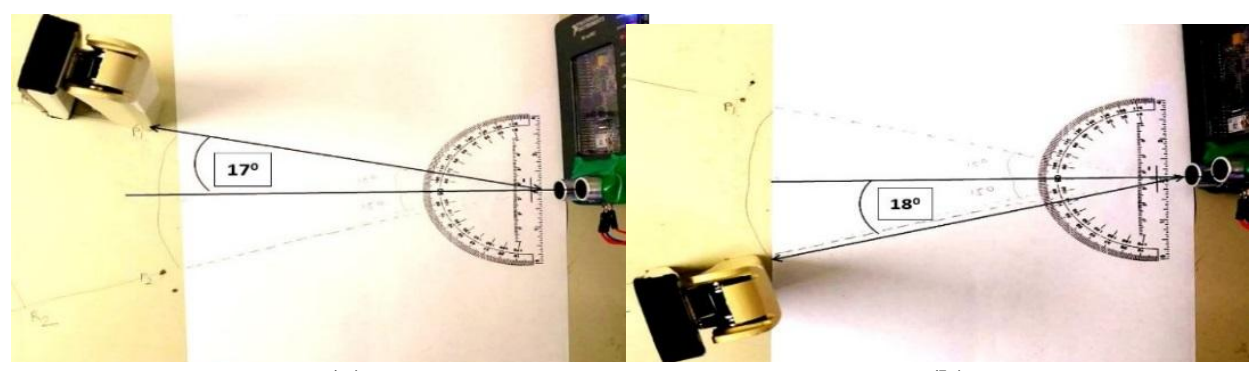

(a)

(b)

Figure 24. Angle of detection for HC-SR04

Figure 24 shows the angle measurement set up for single sensor system. After carrying out number of experiments, the average value for both of the measurements was calculated. For a right hand side obstacle, the average angle of detection was found to be $17^{0}$ (fig 24.a). For left hand side obstacles, it was $18^{0}$ (Fig 24.b).

\subsubsection{Pulse width variation}

We also verified the change in the received pulse width depending upon the distances. For a larger distance, we get a larger pulse width where as it is reduced as we move closer to the obstacle.

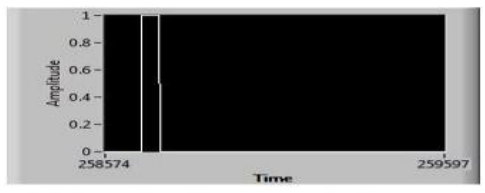

Pulse for distance $1.63 \mathrm{~m}$

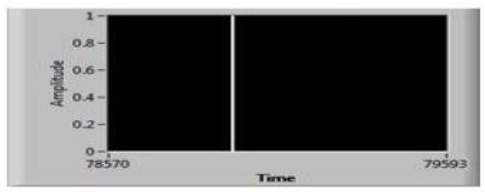

Pulse for distance $0.09 m$

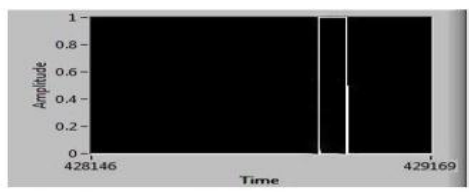

Pulse for distance $2.91 \mathrm{~m}$

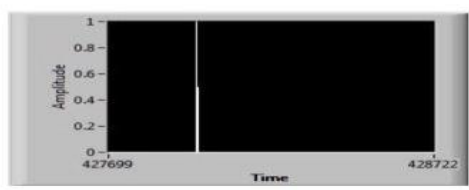

Pulse for distance $0.02 \mathrm{~m}$

Figure 25. Pulse width variation 


\subsection{Three Sensors System:}

As we calibrated our system for a single sensor operation, we can move further towards our aim to cover more area to detect obstacles. So as an intermediate state, we decided to implement this system for three sensors which is capable of detecting obstacles from three different directions. Considering the angle of detection, we kept all sensors $20^{\circ}$ apart from each other so that they cannot interfere for a shorter distance. Also we plotted the 2D grayscale map for this system.

\subsubsection{Design and operation:}

The Design of three sensor system is as shown below. Figure 26.a is a structure of the system whereas the Figure26.b shows the respective mapping.

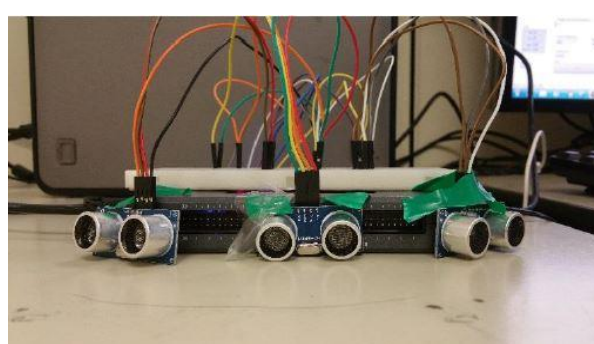

(a)

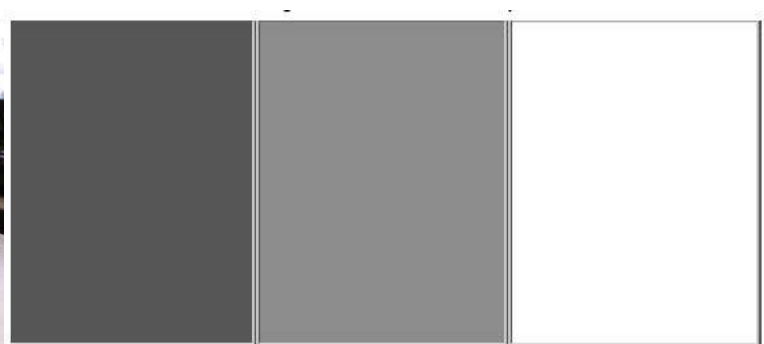

(b)

Figure 26. Three sensor system and mapping

In Figure 26we can see three sensors which are placed $20^{\circ}$ apart from each other. Middle sensor can give the information about the presence of obstacle in center zone. Whereas the left and right sensor can give information about the obstacle in respective zone.

\subsubsection{Mapping:}

Mapping of the obstacles is as shown in Figure 26. From the study we have done, we can conclude that the obstacle towards left hand side is the closest one. It can cause a damage to the user. The center sensor gives a moderate reading indicating that object is comparatively at larger distance than the left side. Right hand side sensor shows no reading, indicating that the right hand zone is safe for the navigation.

On the basis of the successful execution of the three sensor system, it is clear that as we increase the number of sensors, we can extract more information from the 
environment and which can ensure the safe navigation for the user. Keeping this aim in mind, we moved towards the nine sensor system, which is capable of detecting obstacles from nine different positions.

\subsection{Nine Sensors system:}

We studied a single sensor system. Based on the data, we also built a three sensor system. Though our aim was to use a minimal hardware system, a three sensor system is not sufficient to get an approximately correct information about the obstacles in the path. System fails to detect the head level and ground level obstacles. Hence we need to add more sensors to the system to have prime components.

\subsubsection{Design and operation:}

Keeping this goal in mind, we implemented a $3 \times 3$ sensor array system. It can be referred as 'Nine sensor system'. Sensors are placed in a 3x3 array, as in the matrix form. We have initially kept sensors in only one plane to check the interference caused by each sensor. The design of nine sensor system is as shown in Figure 27.

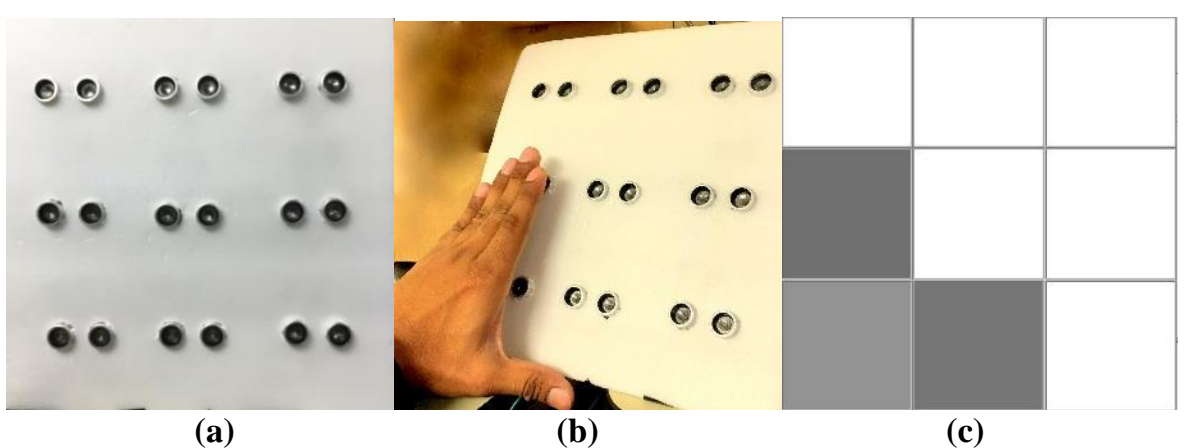

Figure 27. Nine sensor system and obstacle detection

The structure of nine sensor system is as shown in Figure 27a. Each of the sensor is placed at the distance of $10 \mathrm{~cm}$ from the adjacent sensor. The operation of the system can also be tested by placing a hand nearer to the sensors (fig 27b). We can see that the map indicates the possible location of object in the system (fig 27c). It provides a better resolution than a three sensor system. However this system also undergoes interference problem as discussed further. 


\subsubsection{Limitations of the system:}

System faces a large amount of interference because of the spacing and direction of the sensors. It is capable of detecting obstacles at very closer distance, but it fails when we need to find obstacles from larger distance. This is caused by the over lapping of two or more detection zones at the larger distance. This can be explained with the help of figure below,

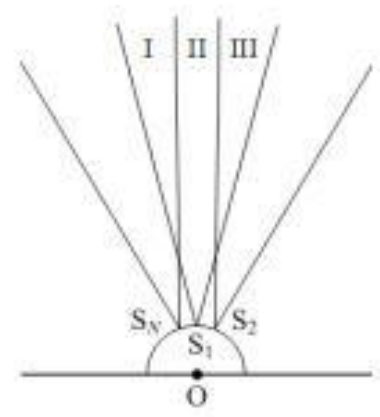

Figure 28. Regional Overlap of sensors [3]

In Figure 28, we can see a three sensor system. While considering overlap, we are considering a horizontal array of three sensors. It is because the ultrasonic sensor has very small vertical detection angle. So vertical sensors are very less prone to the interference.

Now as shown in figure, each of the sensor is associated with the detection zone. The sensor which is at left side of the system has its own detection zone. The sensor on the right hand side also has its detection zone. Now the detection zone of the center sensor is overlapped with the left as well as right hand side sensor.

Zone I represents the overlap between left and center sensor. Zone III represents the overlap between right and center sensor. Where zone II is detection zone for central sensor alone. Any point before the interception of the individual zones is responsible for a desired operation. Within this range, system is capable of detecting and differentiating obstacles. When we move away in overlapped zone, we may not get the accurate results for multiple obstacles. Suppose there is an obstacles in zone I, then it can be detected by both of the sensors. Whereas the sensor will fail if multiple obstacles are placed in the same zone. It cannot detect multiple obstacles which are 
closer to each other. So even if our system is capable of detecting nine different points, it cannot provide a reliable operation to the user. System is giving accurate results for the object which are placed within the range. This feature can be useful in shape detection where the components of a same objects are needed to be extracted.

\subsection{Modified Nine Sensor System:}

A modified nine sensor system is the solution over the nine sensor system. The possible interference caused by adjacent sensors can be eliminated by keeping sensors inclined to each other. Hence it can improve the detection zone of the system. Our system is considering obstacles from directions which can give us the prime information about their existence. Design of the system is explained below.

\subsubsection{Design and operation:}
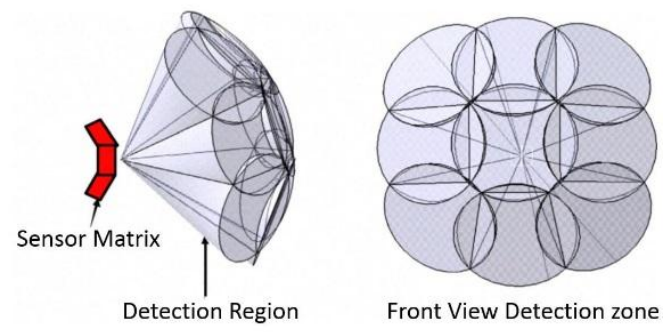

Figure 29. Detection zone for the system [2]

The array of $3 \times 3$ sensors in modified form can be shown as in Figure 29.Each sensor is placed $20^{\circ}$ apart. We can define it in terms of rows and columns. We have upper row, center row and lower row. Similarly we have right column, center column and left column. Upper and lower rows are $40^{\circ}$ apart from each other. Similarly right column and left column are $40^{\circ}$ apart from the center column. The $40^{\circ}$ angle ensures that there is no interference in between two adjacent sensors. Once all the parameters are fixed, we built a system which can be tied around the body with ease. 


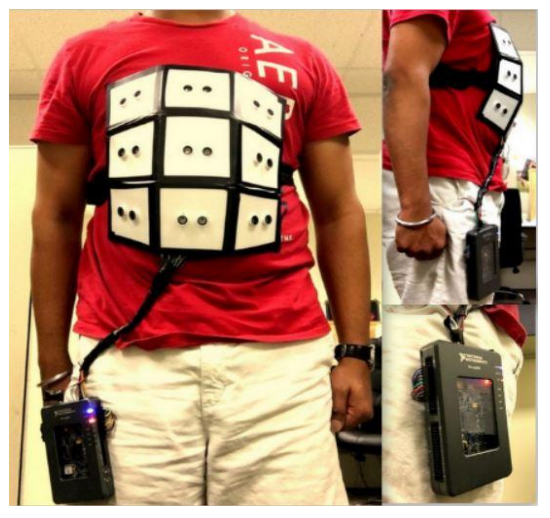

Figure 30. Implementation of wearable system

Figure 30 represents the implemented wearable system. The material used to build the system is very light in weight and durable. Sensors are placed as per the specified format in Figure 29. Wire connections from all the sensors are tied together in a single channel to occupy small space. MyRIO can be tied or can be kept in the pocket. Also we have some modifications over our design in RIO. Depending on the power specifications, the power supply to Rio is provided with the help of the batteries. Also the RIO is connected to the LabVIEW platform through Wi-Fi connection. So far we have covered most of our goals to make system portable, wearable and wireless. Now in order to make our system reliable, we must perform a set of experiments which can detect bunch of obstacles form the sensing plane.

\subsubsection{Validation of the design:}

We carried out number of experiments by creating some scenarios. For each of the scenario, a two dimensional grayscale map was plotted. Primarily all the measurements are based on the stationary state of the user. We also have considered moving state of the user which we are going to explain from the figures.

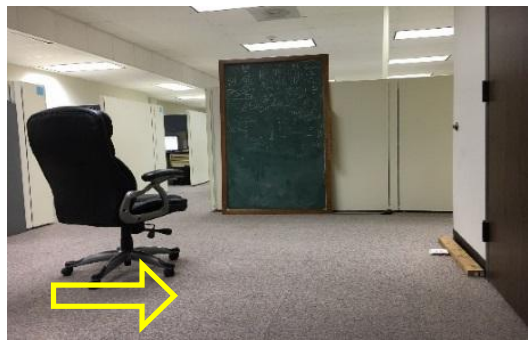

(a)

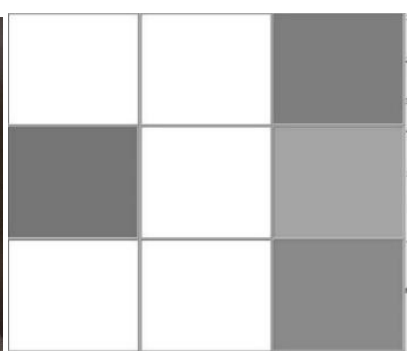

(b)

Figure 31. Scenario 1-RHS detection 
In scenario 1, we have placed a chair towards the left hand side of the user (fig 31a). And there is a door towards the right hand side of the user. It can be seen that the left center block is giving us a grayscale value (fig $31 \mathrm{~b}$ ). This block is associated with the existence of the chair. Whereas the top and bottom sensor from the same side does not give any reading indicating absence of obstacle. Right hand side sensors are giving a grayscale value representing an object on right hand side. Rest of the area is safe for navigation.

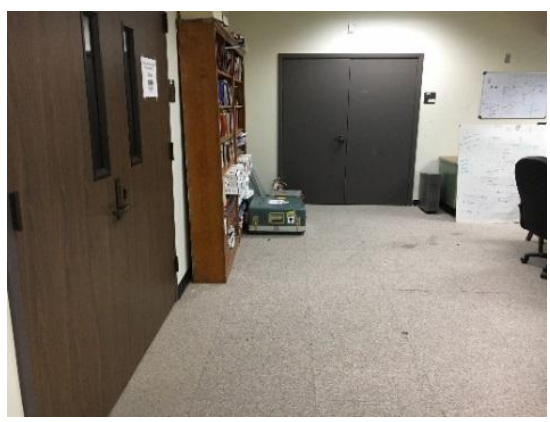

(a)

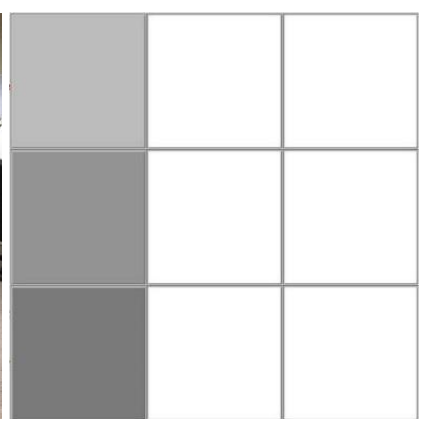

(b)

Figure 32. Scenario 2-LHS detection

In scenario 2, we have only a wall on left hand side (fig 32a). So we can see in the map that we have grayscale values on the left hand side of the map and these values are mainly because of the wall (fig $32 \mathrm{~b}$ ). Whereas the for no grayscale value, we can define the area to be safe.

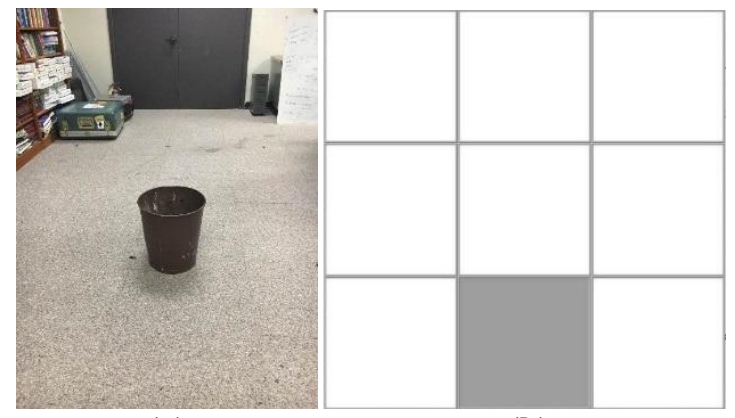

(a)

(b)

Figure 33. Scenario3- Ground level detection

In scenario 3, we placed an obstacle at the bottom level on the ground (fig 33a). This was necessary to ensure the functionality of bottom sensors. As you can in Figure $33 \mathrm{~b}$, bucket can be completely spotted using the map. The grayscale value at the 
center bottom box represents the bucket. Where rest of the boxes indicates the safe path for navigation.

In all the above experiments we have considered stationary motion of the user. In order to have a real time operation, we have now considered the walking state of the user. Also we are introducing multiple obstacles in the path of the navigation. As the walking state of the user is risky for him to navigate in indoor as well as outdoor, we need to test the system for moving state as well.

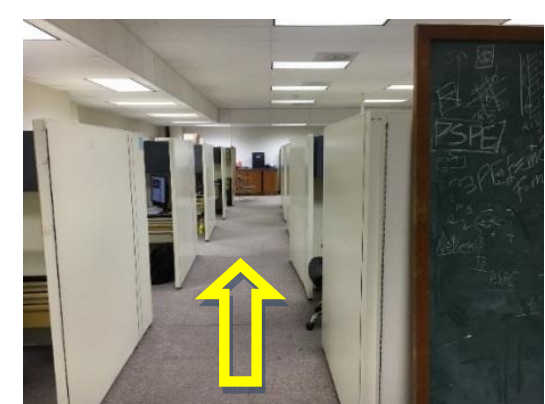

(a)

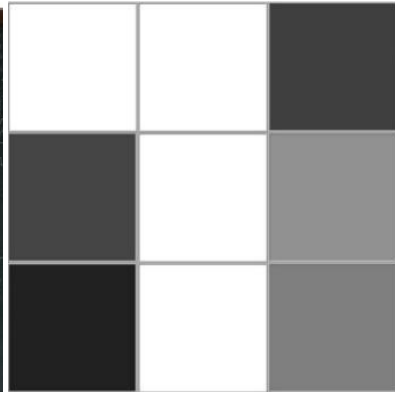

(b)

Figure 34. Scenario 4- walking state with multiple objects

In scenario 4, we made user to pass through a passage which can help him to walk without any collision. The left hand side of the system has compartments and the right hand side of the user has a board (Fig 34a). We can also see that the right hand side object is taller than the right hand side object. From Figure 34.b, if we look at the map, the right hand side represents the board which is on right hand side. Also, on left hand side, two grayscale blocks represent the compartment. The middle white boxes indicate the safe path for the navigation.

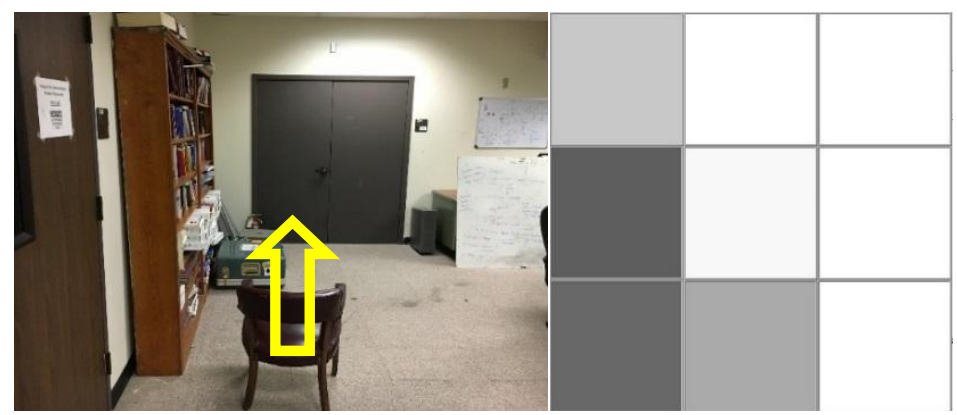

(a) (b)

Figure 35. Scenario 5- walking state with LHS and center detection 
We have a wall on the left hand side and a chair placed in front of the user. The user is in walking state. The left hand side boxes show a grayscale image representing the wall. Also the center bottom box represents the chair. Now in this case the height of the chair is important. Because of the small vertical angle, rest of the sensors in the same column did not given any notification about the object. The rest of the boxes which did not show grayscale represent safe zone.

\section{- Object detection in motion:}

So far we have considered the detection of obstacles when the user is in stationary state. Now we are considering the user in the motion in order to check the accuracy. The walking speed is considered to be typical and fast. Typical speed is about forty steps in a minute. Fast motion is considered to be a one feet per second

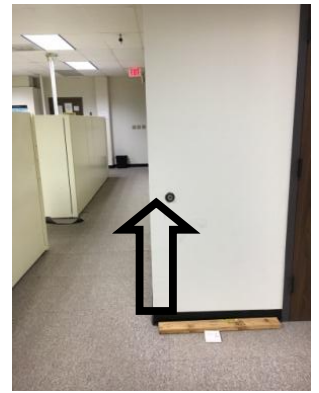

(a)

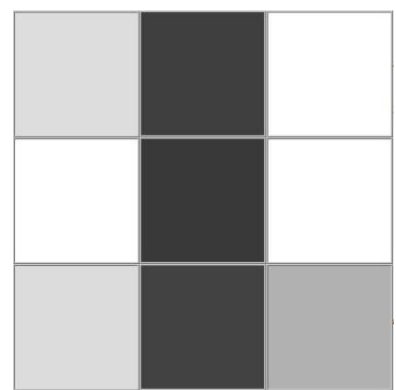

(b)

Figure 36. Scenario6- walking state object detection

Figure 36a shows the scenario where a door is at center. It successful detected the object when it was in slow motion (Fig 36b).

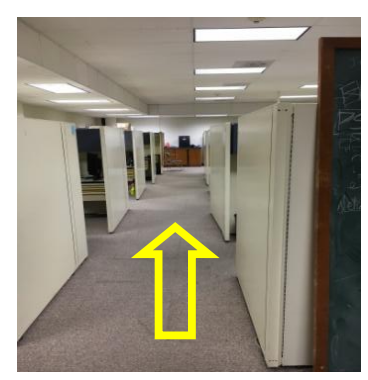

(a)

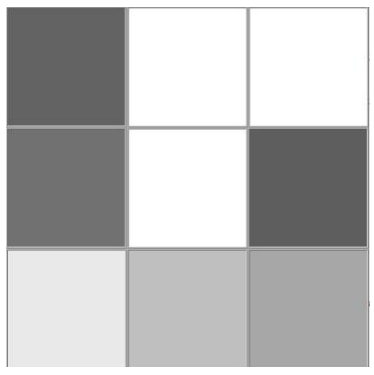

(b)

Figure 37. Scenario7- Walking state object detection

Figure 37a shows the scenario where compartments are on the LHS and RHS allowing user to pass through the passage. It successful detected the object when it was in slow motion (Fig 37b) 


\subsubsection{Three dimensional mapping:}

As we have discussed earlier about the three dimensional mapping which we are going to implement in this system. This three dimensional map will be in terms of the projections. To calibrate our system for 3D mapping, we have set a boundary for $2 \mathrm{~m} \times 2 \mathrm{~m}$. Ideally this area can be the safe navigation area for the user. It is sufficient for the user to know about the obstacle in his path before $2 \mathrm{~m}$. Each side of square is divided into three parts, so we have 9 boxes for 9 sensors. 3D mapping can be represented as below,

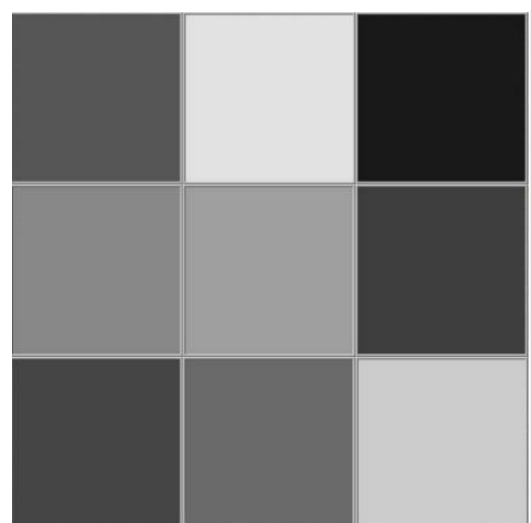

(a)

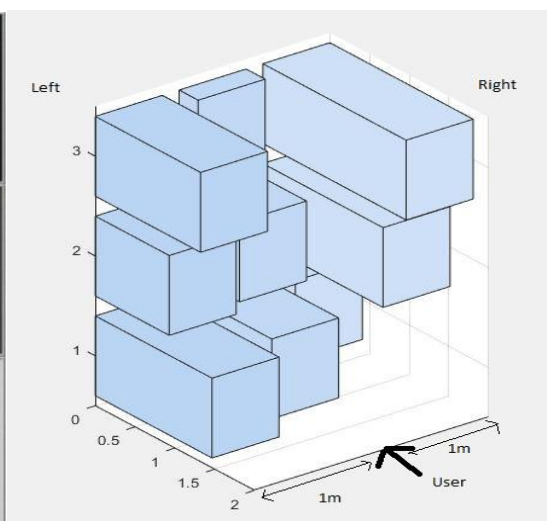

(b)

Figure 38. Three dimensional mapping using projections

As you can see in Figure 36, we have one-to-one projection for each box in grayscale image. User is assumed to be standing exactly at the middle of the edge of box (Fig 36b). The top right corner shows low grayscale value that means we have an obstacle closer to the user (Fig 36a). If you map the same box in 3D view, you can actually imagine the right top corner with a projection closer to the user. Similarly the center top box has maximum grayscale value, which means obstacle is farthest. In 3D map we can see that the box has least project indicating that the object is at the farthest distance as compared to other objects.

\subsection{Advantages of the system:}

- External power supply makes system portable. We have used dry cell batteries which are capable of providing $9 \mathrm{~V}$ supply with the power consumption of $14 \mathrm{~W}$. However in future, these batteries can be replaced by the more efficient batteries. 
- System further supports the portable nature by using a Wi-Fi module. The LabVIEW platform remains connected with the RIO through this wireless connection.

- RIO also offers Internet Protocol. This makes it accessible through the internet connection from anywhere we want.

- RIO provides very flexible operation because of the FPGA. It implements a parallel processing which makes the deployment very fast and simple.

- Sensors are very light in weight and provide an accuracy of $99.2 \%$.

- System offers a very flexible functionality if we want to add more hardware to it.

- Wearable module is very light in weight and can be tied around the body without any trouble. Also RIO is very handy and occupies very less space.

\subsection{Limitations:}

Like all the existing system, our system also suffers with some limitations to it. They can be explained as follows,

- Power consumption is main problem with our system. FPGA modules present on the board consumes more power than required.

- Wearable module occupies more area which can sometimes cause a frustration.

- Though MyRIO is providing SoC operation, we have some components which are never used, consumes more power and area.

- The functionality of the sensing module depends on the type of surface it is facing. Critical angle of reflection is a major issue. When the angle of incidence is greater than the critical angle, the reflected wave may not come back to the receiver.

- Also if the surface of the object is rough then it may not provide the exact reading. 


\subsection{Future work:}

The base experiment was up to the mark, hence there is a scope for the future modifications in order to make the system more reliable, more stable, and compact and power efficient. We can suggest following modifications for the future work.

- Sensor module- The sensors we are using were only for the experimental purpose. In future, the size of sensors can play a good role. The sensors with a small size and high directivity will be more efficient. As they will occupy lesser area, we can accommodate more number of sensors in smaller space. More number of sensors will give us the more information about the obstacles.

- Signal Processing- MyRIO was used for experimental purpose as it provides very flexible operation and also offers SoC. We can also use the accelerometer which is present on board, which can be used in fall detection. System can be easily interfaced with GPS which can provide information about positioning. Further, we can find a solution for this as an ASIC. This can provide a dedicated hardware for the system consuming less area and less power but providing a fast operation. Though the data consumed is very less for now, but in future, when we increase the number of sensors, we will be dealing with very high and complex data, hence ASIC design is going to be prominent.

- Output/Indicators- The approach we followed for the mapping is limited for only nine points. With more number of sensors, we can get a better structure for the obstacles in the pathway of the user. Also vibration motors can be of importance. For a blind person, it is impossible to sense the things just by hearing aids. Hence if we map the systems on the body of the user, it will convey much more information to him. Vibration motors can be used to map this around the body. Also we can create a template of maps. So that it can be used globally by other users. 


\section{CHAPTER VI}

\section{CONCLUSION}

Our work mainly focuses on the system which is wearable, portable and capable of providing human assistance. This assistance is for blind people to navigate in the surrounding. System detects obstacles and instructs user to navigate through a safe path. We followed all the basic phases of this project.

Initially we studied the background about the human assistance and obstacle detection. We came across different ways to achieve our goals. This study was sufficient to move further. We also studied the previous as well as existing systems thoroughly, which follow the same path. Considering the drawbacks and flaws in those system, we implemented our system using acoustic sensor array, NI myRIO 1900 and LabVIEW. We discussed about their features. Acoustic sensor are reliable, and convey important information by consuming very less data. NI myRIO provided very flexible operation. Different features of RIO helped us to make system, faster, reliable and portable. Mapping technique is found to be more efficient than existing techniques. For distance measurement, system showed an accuracy of $99.2 \%$. It was capable of detecting obstacles at an angle of $35^{\circ}$.

Thirdly, implementation of the system was successful. System was wearable and less complex. We successfully tested our system for a single sensor, three sensors and nine sensors. We modified our system to remove all flaws in previous system. We tested our system for stationary state as well as moving state. We get all results nearly accurate, which mapped the objects successfully. System suffered from issues like nature of surface of obstacles, power consumption and area. Overall performance of the system was satisfactory and offers a scope for future work.

It is human tendency to always walk in the way of excellence. Day by day, technology is getting better and better. Human is trying to overcome all the problems he is facing within day to day life. This work was an attempt to be a part of the same flow. In future, we can hope to see a better version of such systems. 
Texas Tech University, Ashish Patankar, May 2016

\section{CHAPTER VII \\ REFERENCES}

1] D. Y. K. Sampath; G. D. S. P. Wimalarathnec, " Obstacle classification through acoustic echolocation", International Conference on Estimation, Detection and Information Fusion (ICEDIF) Pages: 1 - 7, Year- 2015

2] K.H.Yu; M.J.Yoon; Gu-YoungJeong, "3D detection of obstacle, distribution and mapping for tactile simulation" IEEE International Conference on Mechatronics. Malaga, Spain, Pages: -, Year- 2009.

3] S.Kim; H.Kim, 'Simple and Complex obstacle detection using an overlapped ultrasonic sensor ring",12th International Conference on Control, Automation and System (ICCAS), Pages: 2148 - 2152,Year-2012

4] J.h. Lee; K. Kim; S. C. Lee; B. S. Shin, "Smart backpack for visually impaired person", International Conference on ICT for Smart Society (ICISS), Pages: 1 4, Year-2013

5] C.Nadee; K.Chamnongthai, "Ultrasonic array sensors for monitoring of human fall detection", 12th International Conference on Electrical Engineering / Electronics, Computer, Telecommunication and Information Technology (ECTICON), Pages: $1-4$, Year-2015

6] W.C.S.S.Simões; V.F.deLucena,"Blind user wearable audio assistance for indoor navigation based on visual markers and ultrasonic obstacle detection", IEEE International Conference on Consumer Electronics (ICCE), Pages: 60 - 63, Year-2016

7] R. Pyun; Y. Kim; P. Wespe; R. Gassert; S. Schneller, "Advanced Augmented white cane with obstacle height and distance feedback", IEEE International Conference on Rehabilitation Robotics (ICORR), Pages: 1 - 6, Year-2013

8] http://www.micropik.com/PDF/HCSR04.pdf Ultrasonic Sensors HCSR04

9] http://sine.ni.com/nips/cds/view/p/lang/en/nid/211694 
10] Edward R. Dougherty, Roberto A. Lotufo, "Hands-on Morphological Image Processing", SPIE Press, 2003

11] http://www.theatlantic.com/technology/archive/2013/06/this-glove-could-helpdeaf-blind-people-communicate-with-anyone-anywhere/277240/

12] http://wonderopolis.org/wonder/how-does-technology-change-lives/

13] http://inhabitat.com/volvo-unveils-innovative-self-parking-car-concept/

14] http://pointclouds.org/documentation/tutorials/ground_based_rgbd_people_detect ion.php

15] http://www.rf-frontend.de/en/24GHz-radar.html

16] http://proyectojuanchacon.blogspot.com/2015/05/getting-started-with-lidardata.html

17] http://www.wisegeek.org/what-is-a-microcontroller.htm

18] http://www.wikid.eu/index.php/Acoustic_sensor

19] https://ccse.lbl.gov/people/kaushik/papers/AIAA_Monterey.pdf-paper-acoustic

20] http://www.ab.com/en/epub/catalogs/12772/6543185/12041221/12041229/Ultras onic-Advantages-and-Disadvantages.html

21] http://www.ni.com/white-paper/6984/en/--- FPGA

22] http://www.xilinx.com/fpga/asic.htm-ASIC

23] http://tutorial.cytron.com.my/2012/10/11/testing-ultrasonic-ranging-module-snhc-sr04/ 